Mesopotamia J. of Agric.

Vol. (41) No. (2) 2013
ISSN:2224-9796(Online)

ISSN: 1815-316x (Print)

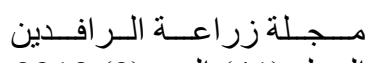

المجلد (41) العدد (2) 2013

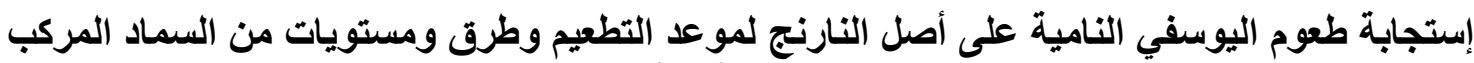
ستاركتشار أكتا أغرو الغوري

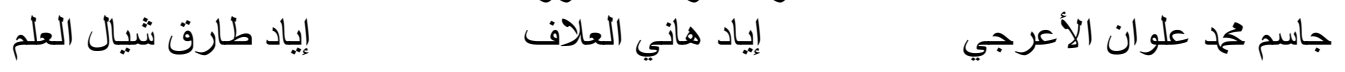

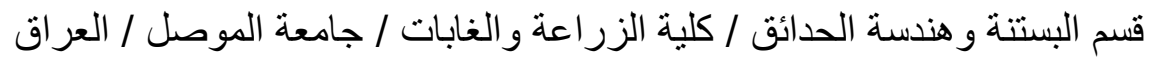

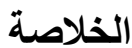

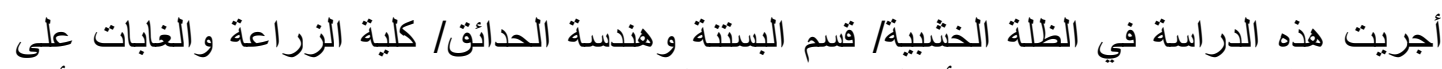

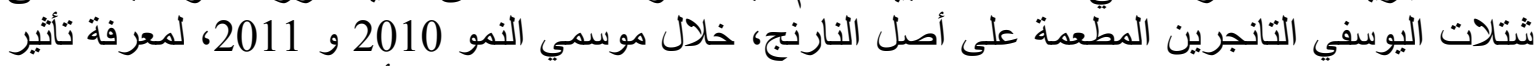

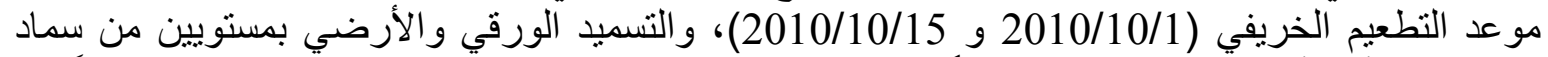

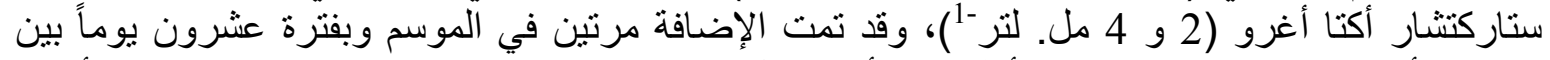

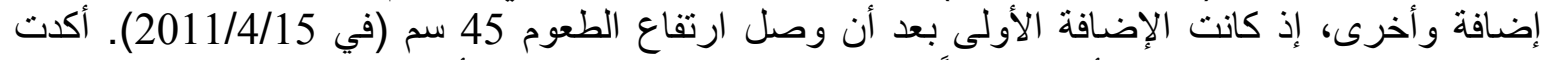

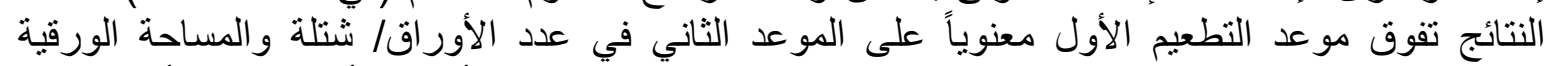

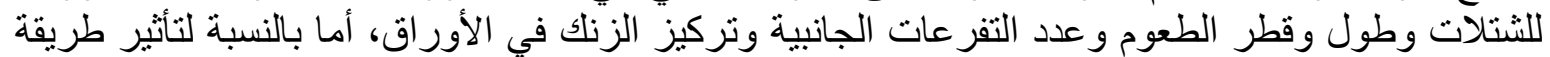

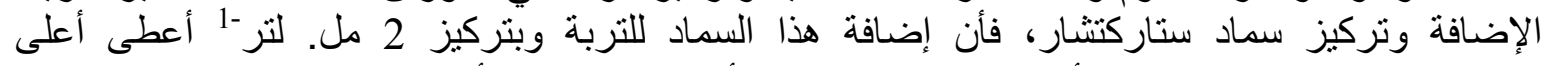

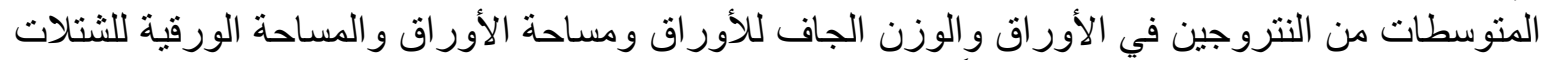

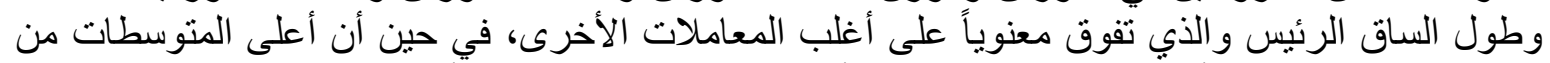

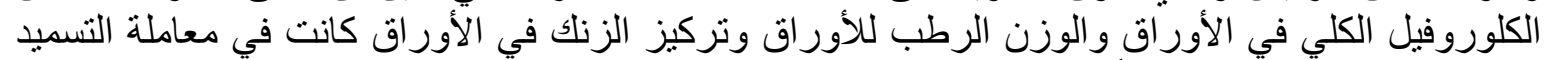

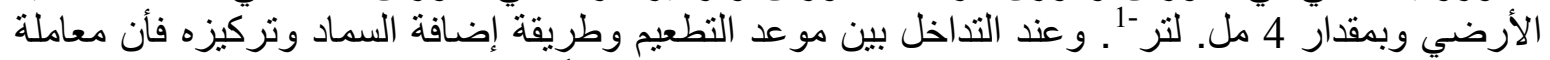

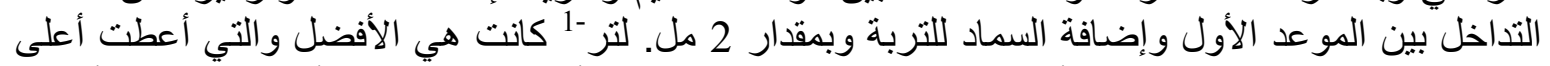

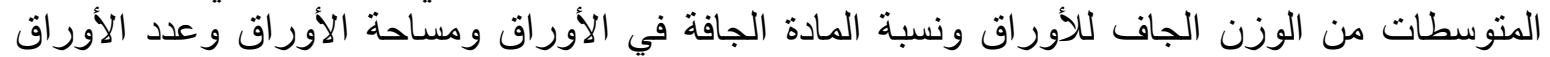

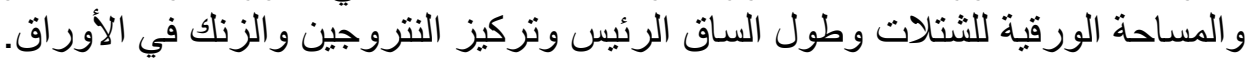
الكلمات الدالة: شتلات اليوسفي. مو عد التطعيم. سماد ستاركتشار.

$$
\text { تاريخ تسلم البحث: 2011/12/18 وقبوله: 2012/3/5 }
$$

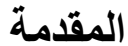

يعد اليوسفي التانجرين من أنواع الحمضيات التي تتبع الجنس Citrus و العائلة

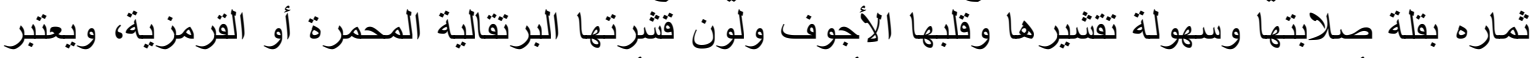

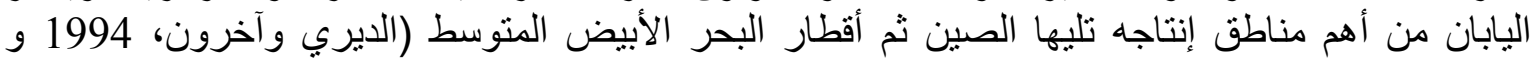

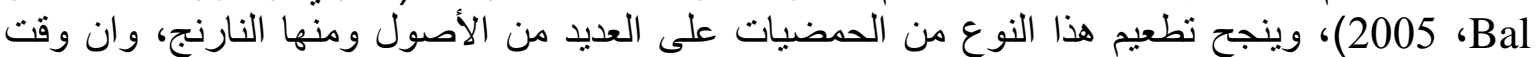

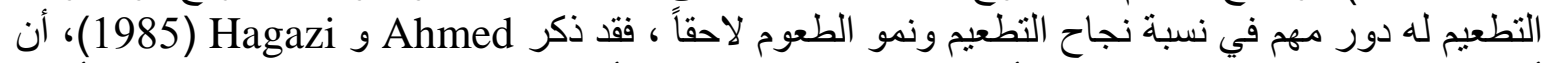

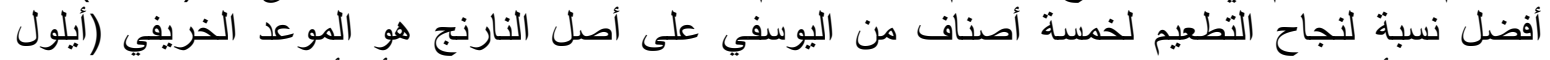

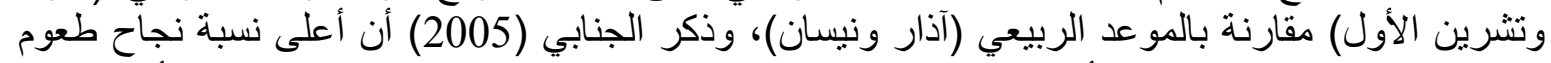

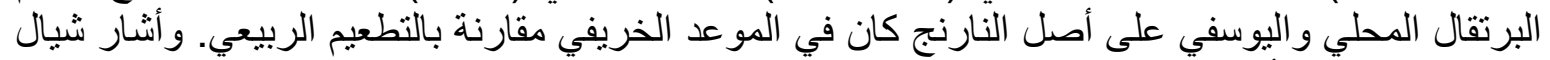

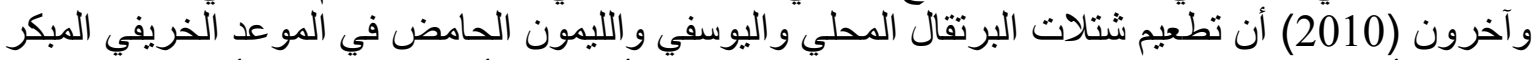

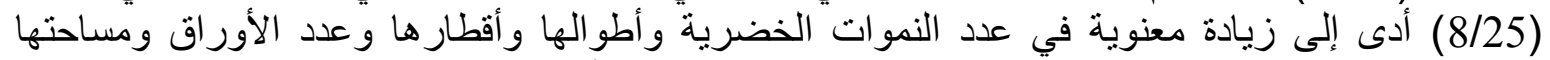

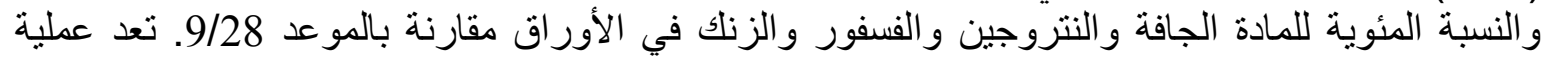

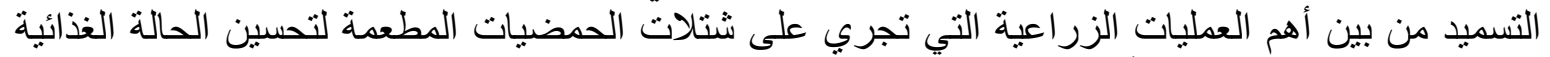

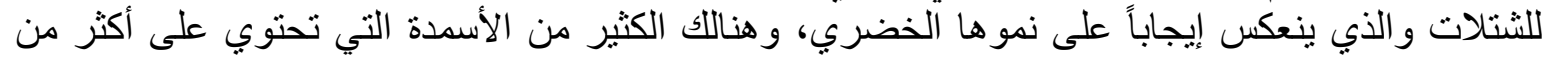

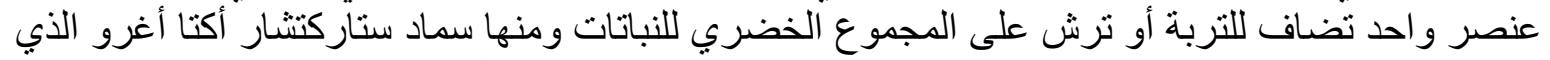

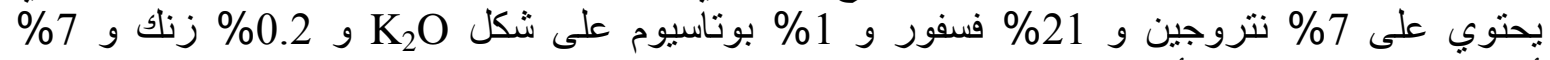

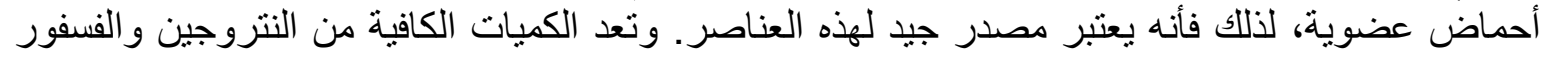

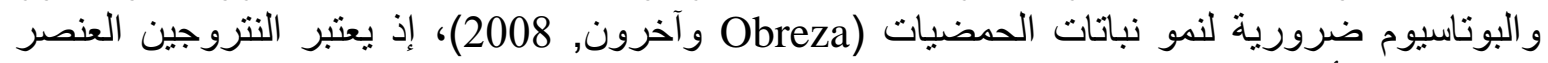

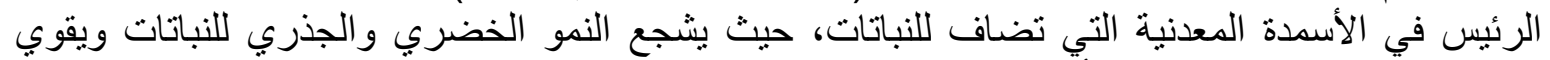

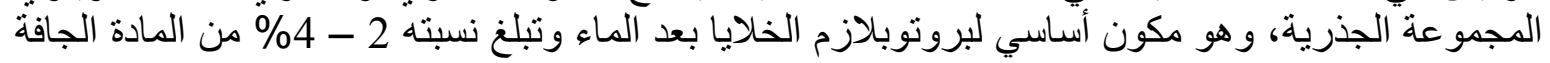


Mesopotamia J. of Agric.

Vol. (41) No. (2) 2013
ISSN:2224-9796(Online)

ISSN: 1815-316x (Print)

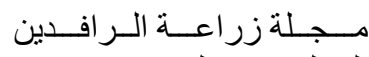

المجلد (41) العدد (2) 2013 الـرافين

للنبات، ويدخل في تركيب المركبات العضوية المهمة مثل الأحماض الأمينية و البروتينات والأحماض النووية

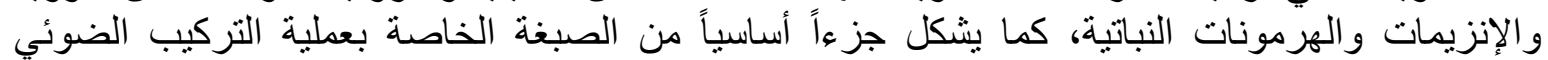

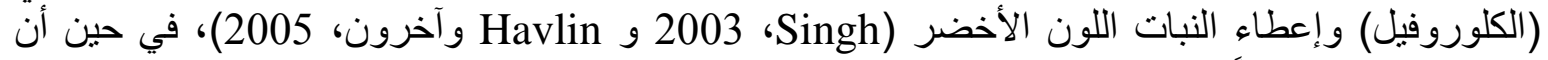

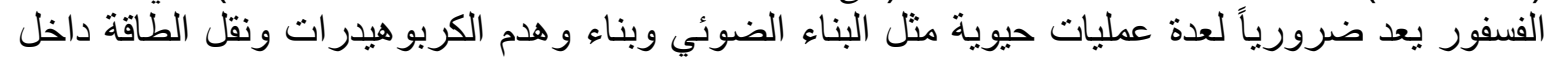

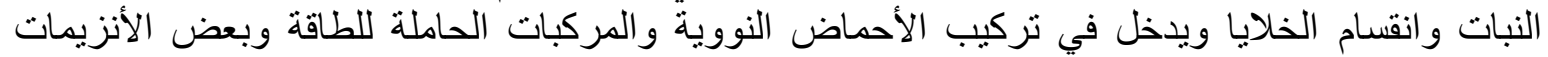

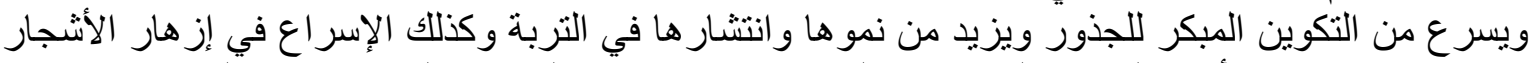

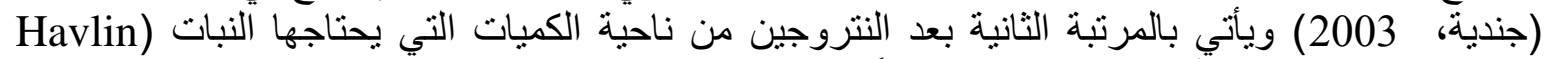

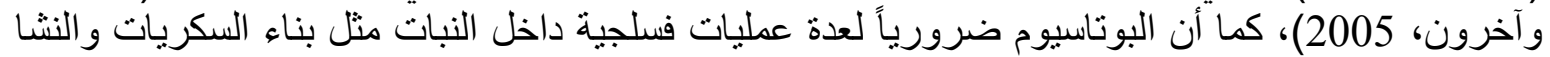

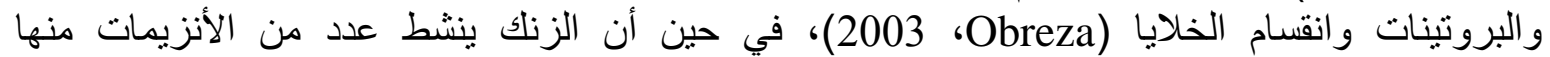
Carbonic anhydrase حماية البروتينات من تغيير طبيعتها، ؤأنزيم Starch synthase الضروري في في الإني تصنيع النشا وتحتاج إليه

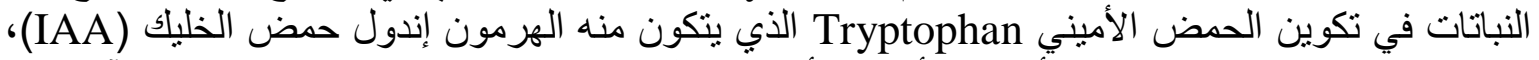

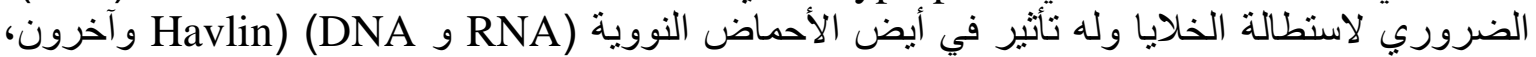

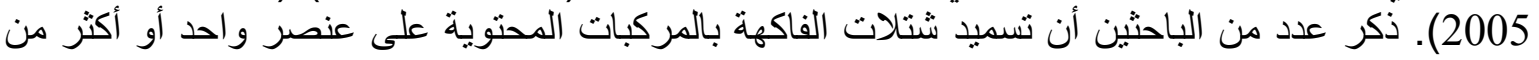

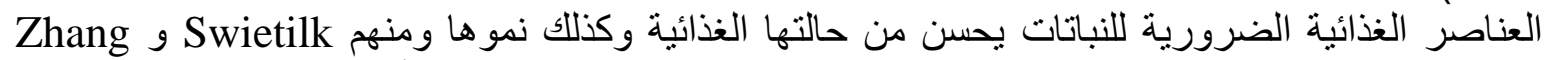

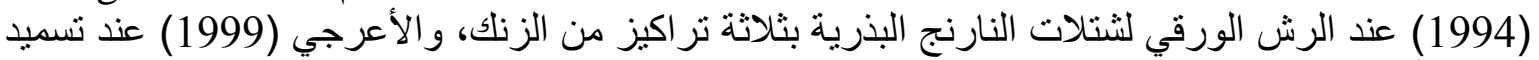

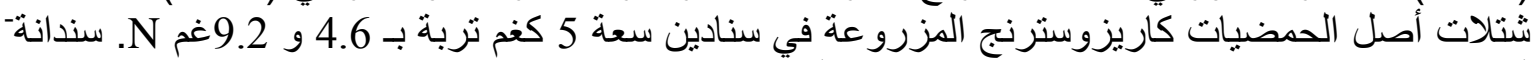

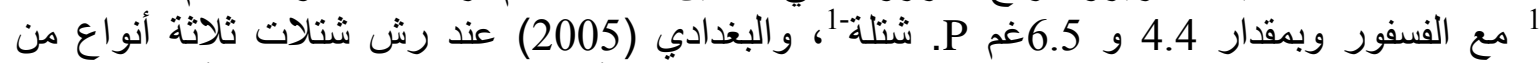

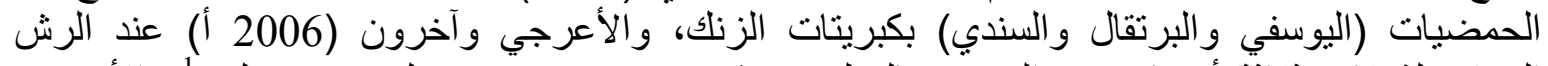

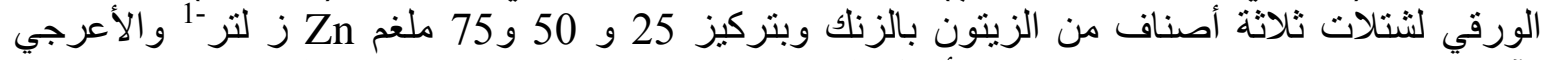

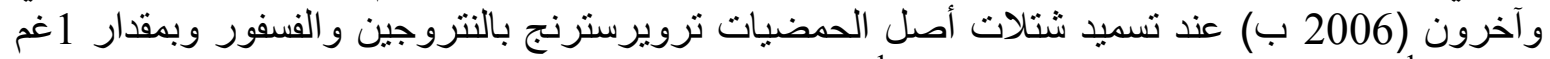

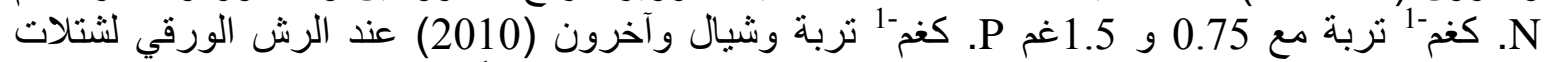

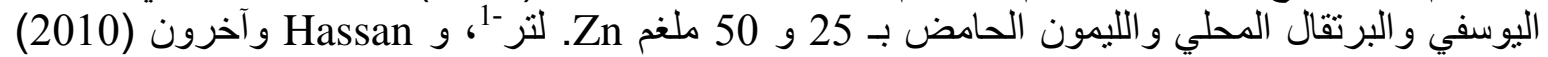
عند تسميد شتلات المانكو بسمادي اليوريا واليوريا فورمالدهايد

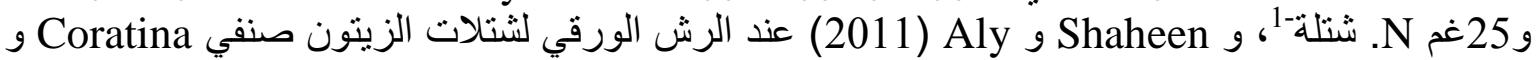
Maraki

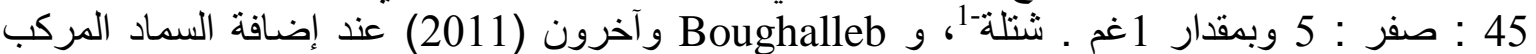
NPK

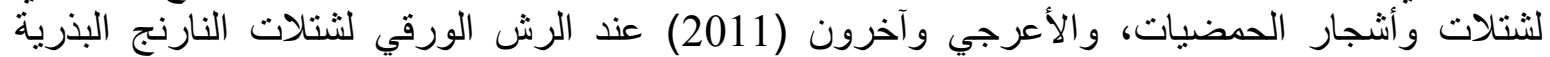

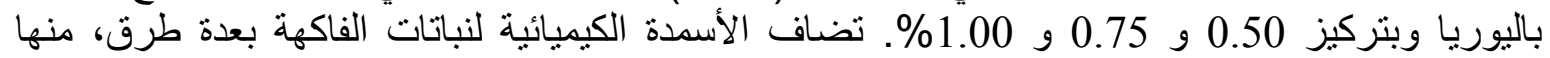

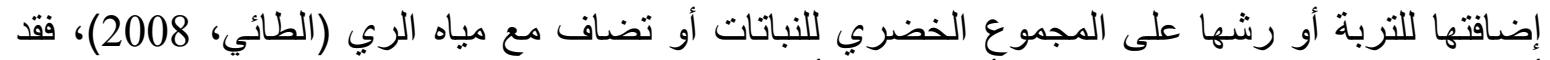

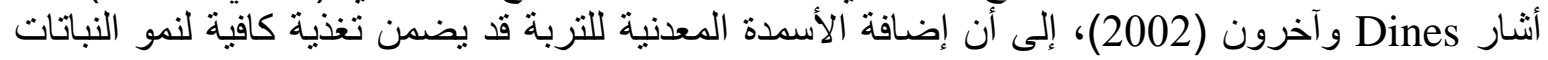
و إنتاجها، ولكن هنالك تخوف من فقد المغذيات عن طريق عملية الغسل التي قد تحصل خاصة في ترب الترب الترن

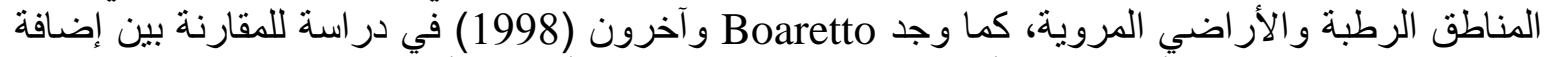

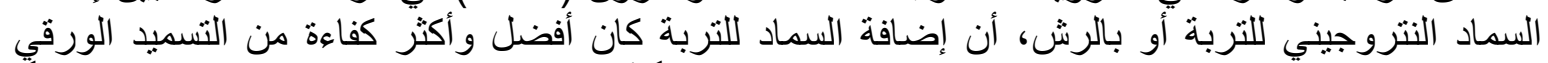

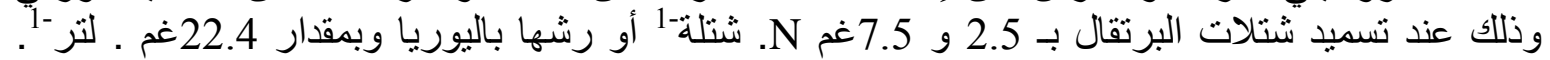

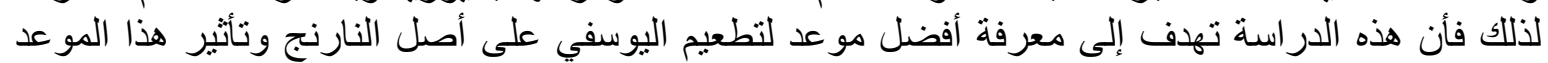

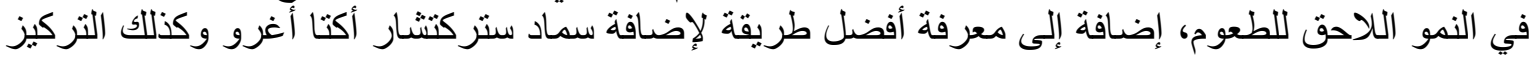
المناسب من هذا السماد للحصول على شتلات من اليوسفي المطعمة الصالحة للزر اعة في المكان المستديم.

\section{مواد البحث وطرائقه}

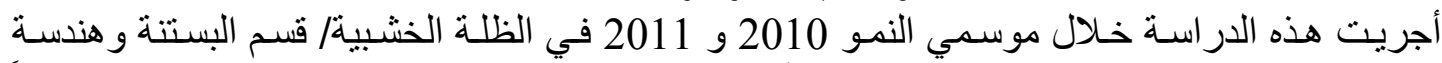

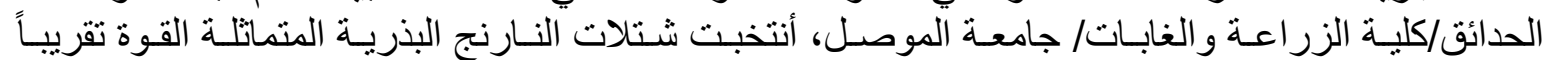

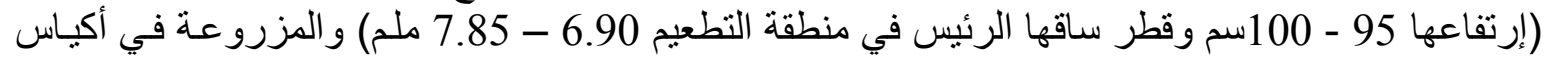

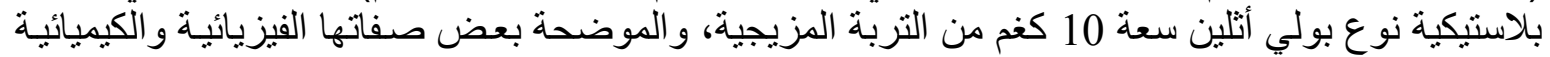
في الجدول (1) و التي أخذت من مشتل قسم البستتة وهندسة الحدائق، وكان التطعيم في خريف عـام 2010 
Mesopotamia J. of Agric.

Vol. (41) No. (2) 2013
ISSN:2224-9796(Online)

ISSN: 1815-316x (Print)

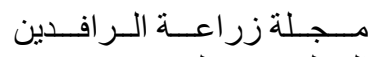

2013 المجلد (41) العدد (2)

وبمو عدين هما: 2010/10/1 و 2010/10 بطعوم اليوسفي التانجرين وتركت الثتلات تنمو في الظلة

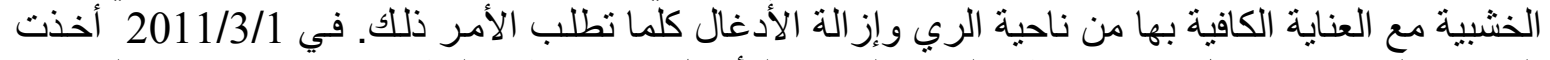

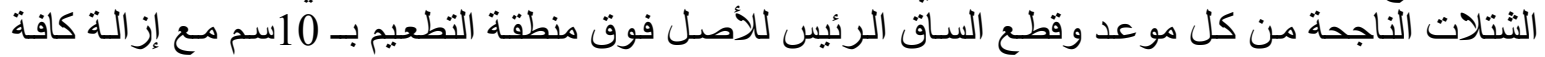

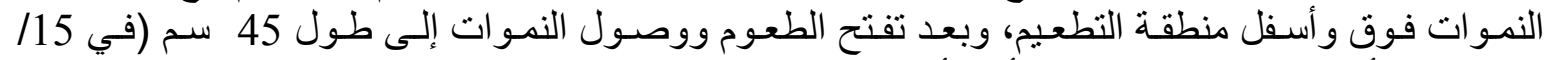

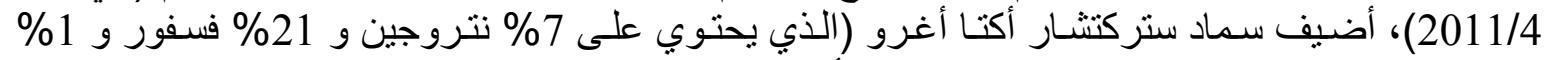

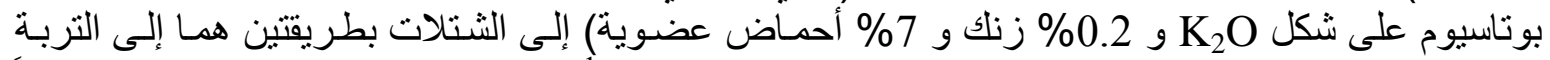

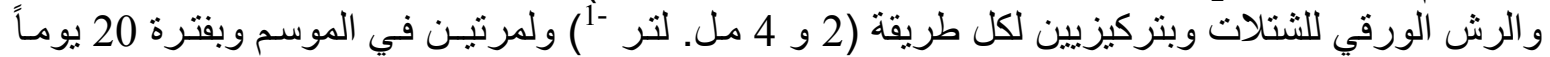

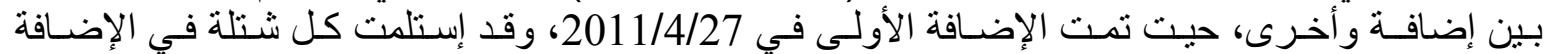

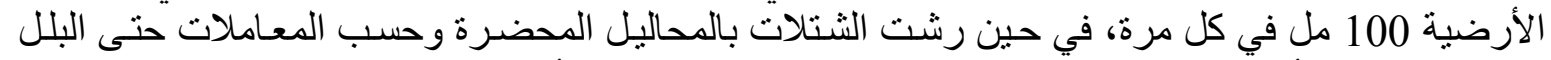

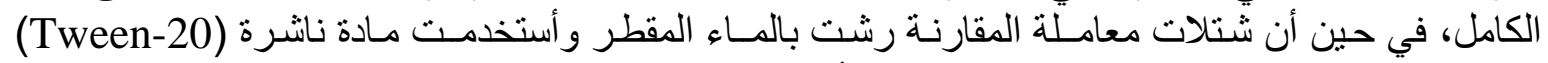
وبتركيز 1.00\% لتجانس توزيع المحاليل على الأوراق.

الجدول (1): بعض الصفات الفيزيائية و الكيميائية للتربة.

Table (1): Some physical and chemical properties of the soil.

\begin{tabular}{|c|c|c|c|}
\hline $\begin{array}{l}\text { القيمة } \\
\text { Value }\end{array}$ & Parameter الصفة P & $\begin{array}{c}\text { القيمة } \\
\text { Value }\end{array}$ & Parameter الصفة \\
\hline 143.9 & (ملغم.كغק-1 ) $\quad \mathrm{CaCO}_{3}$ & 462.55 & 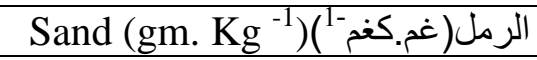 \\
\hline 49.00 & 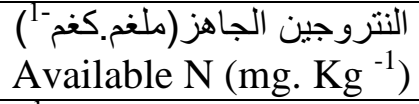 & 306.55 & Silt(gm. Kg ${ }^{-1}$ (الغرين(غم.كغم) \\
\hline 22.00 & 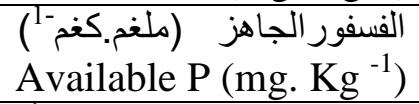 & 230.90 & Clay (gm. Kg $\left.{ }^{-1}\right)\left({ }^{1-1}\right.$ الطين(غم.كغ \\
\hline 130.00 & 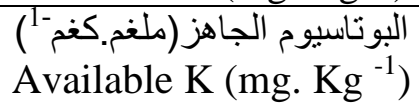 & مزيجية & Soil texture نسجة التربة \\
\hline 0.40 & 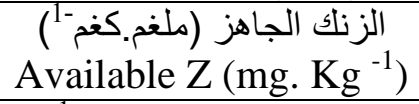 & 17.10 & 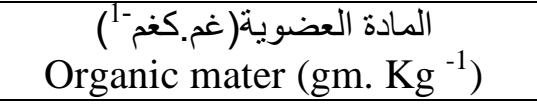 \\
\hline 31.29 & 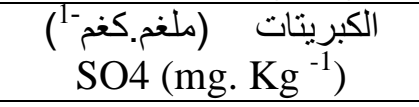 & 1.456 & (dsm.m $\left.{ }^{-1}\right)$ (دسي سيمنز .م-1- EC \\
\hline & & 7.53 & لتربة (pH) \\
\hline
\end{tabular}

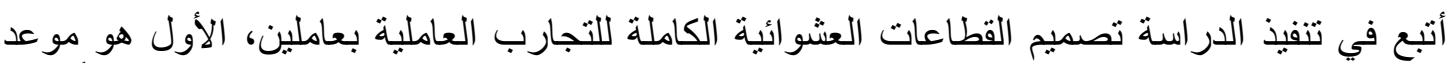

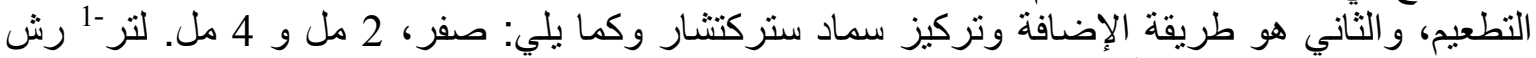

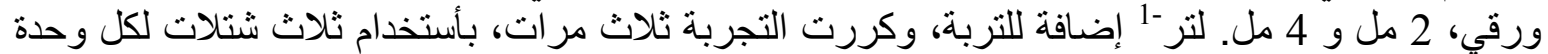

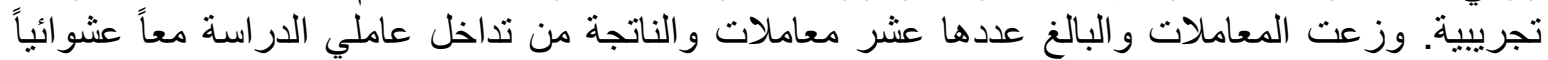
داخل كل مكرر. في بداية شهر آب من العام 2011 تم قياس محتوى الأوراق من من الكلوروفي النيل (وحدة

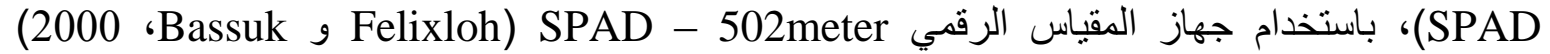

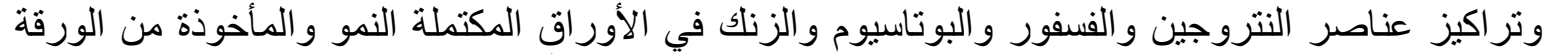

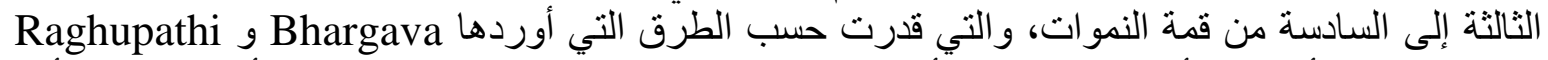

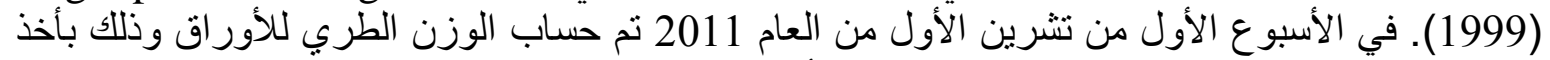

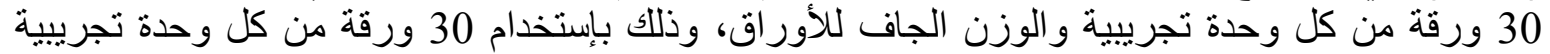

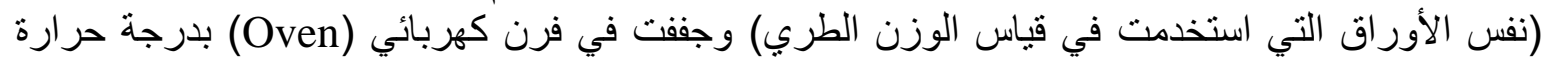

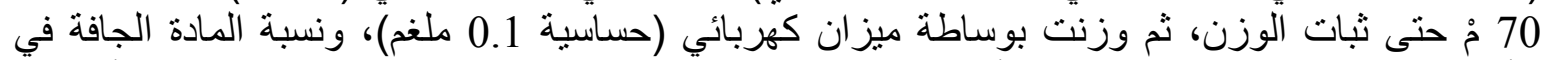

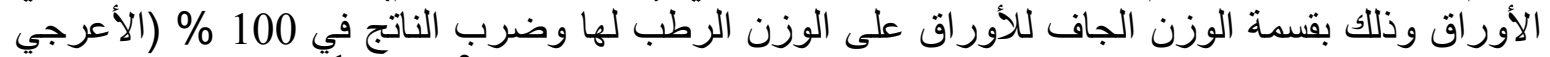

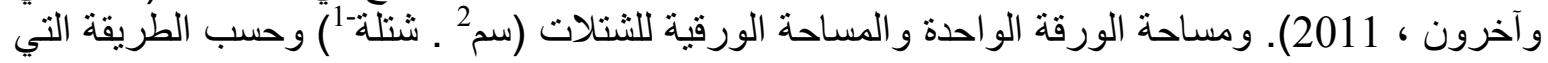

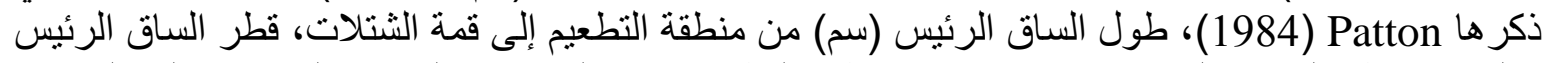

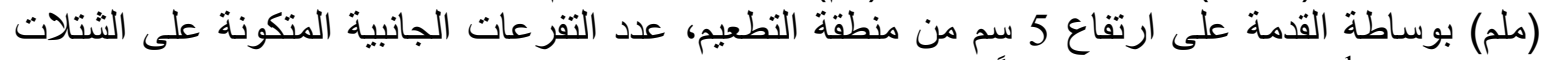

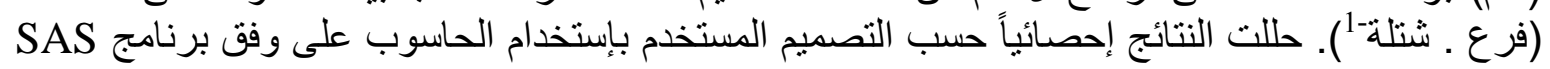


Mesopotamia J. of Agric.

Vol. (41) No. (2) 2013
ISSN:2224-9796(Online)

ISSN: 1815-316x (Print)

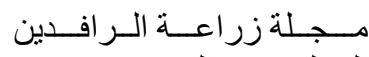

المجلد (41) العدد (2) 2013 الـرافين

\section{النتائج والمناقشة}

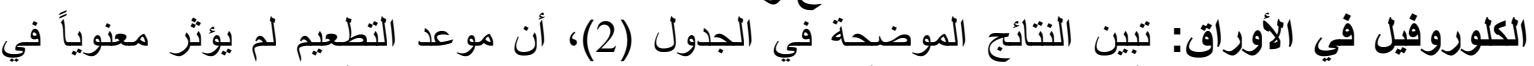

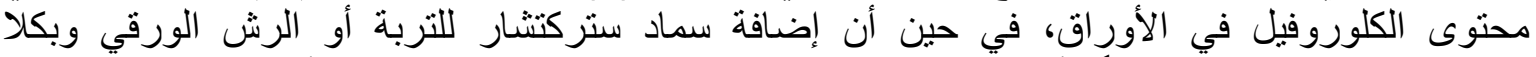

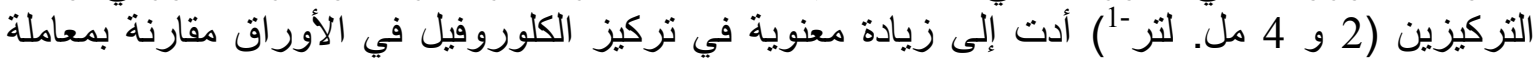
المقارنة، وأن أعلى التراكيز من هذه الصفة كانت عند إضافة هذا المركب للتربة وبكلا فئل التركيزين (2 و 4

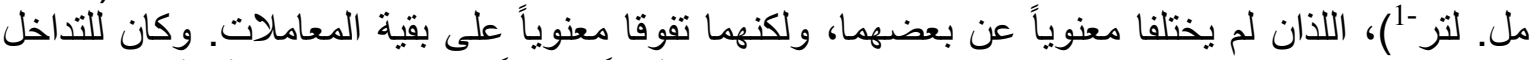

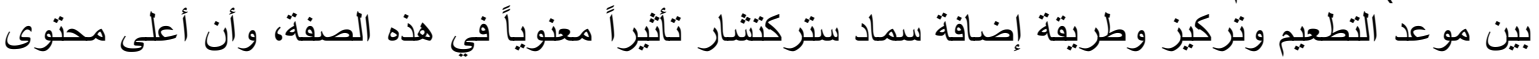

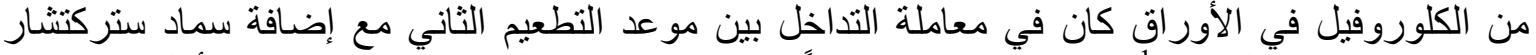

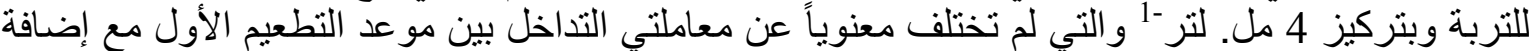

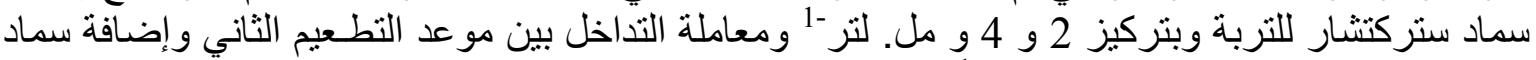

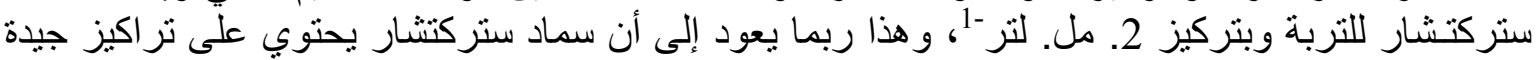

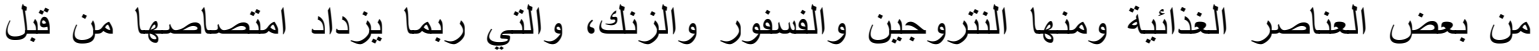

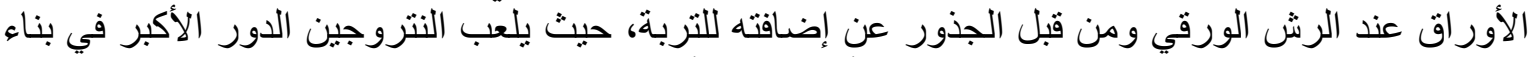

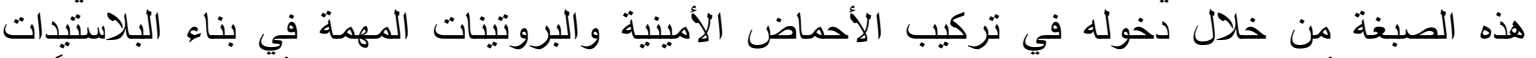

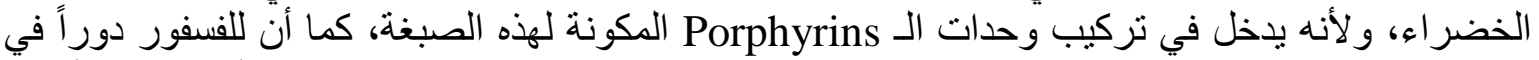

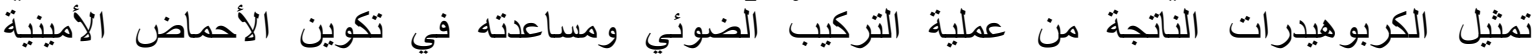

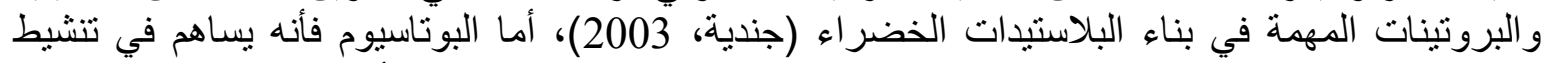

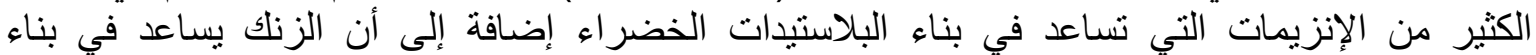

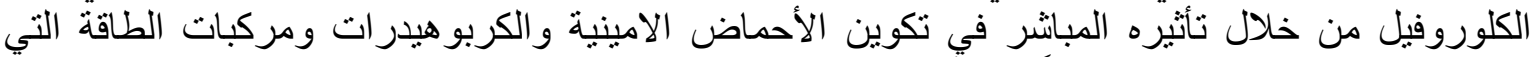

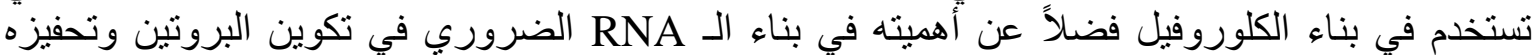

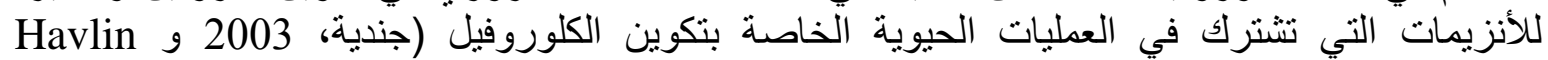
وآخرون، 2005).

صفات النمو الخضري

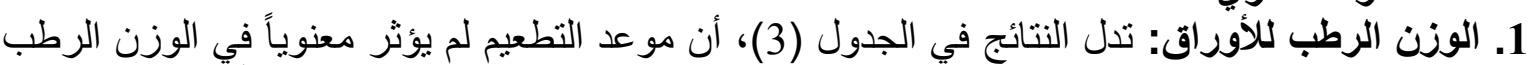

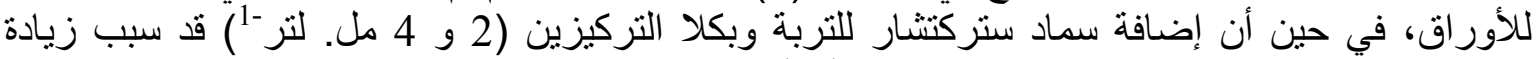

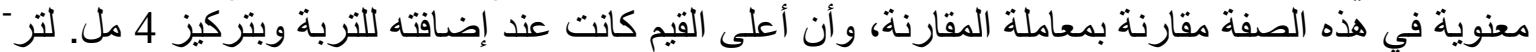

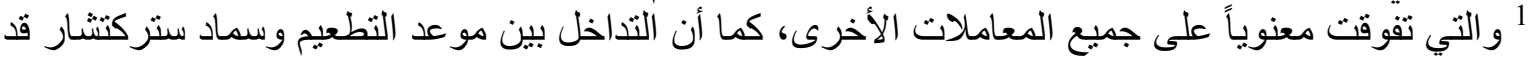

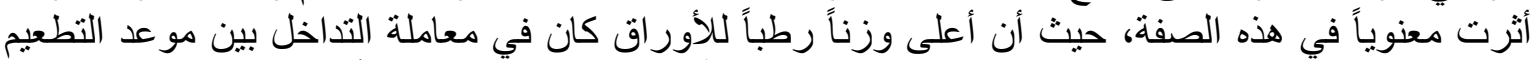

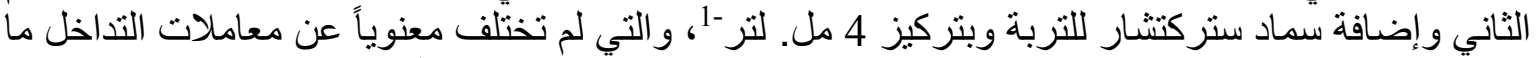

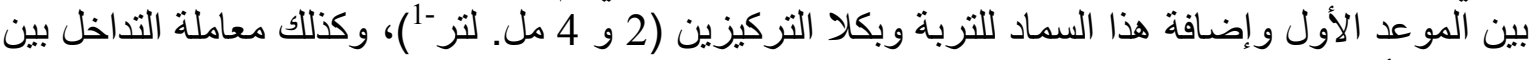

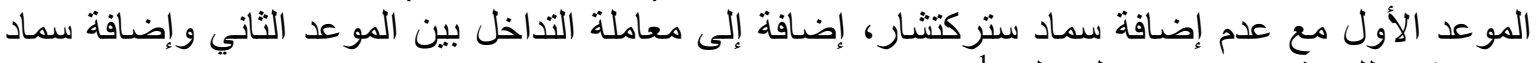

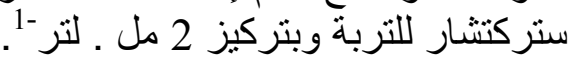

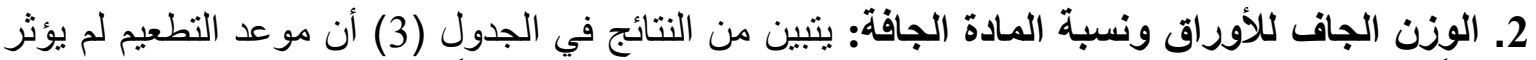

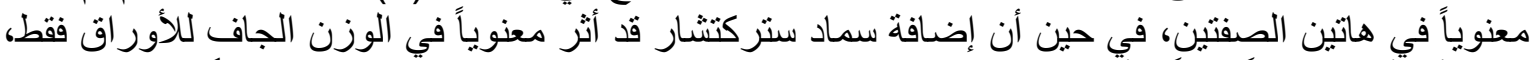

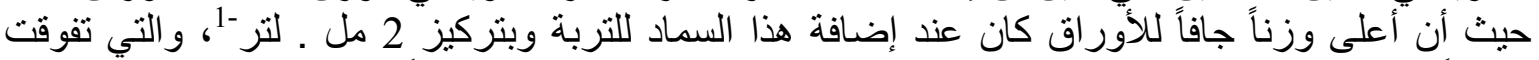

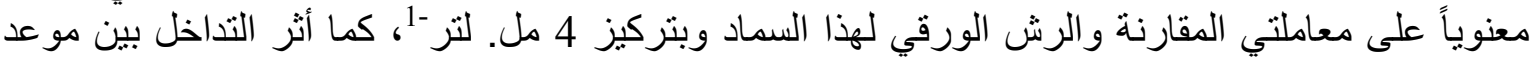

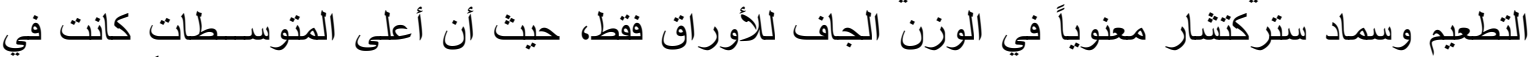

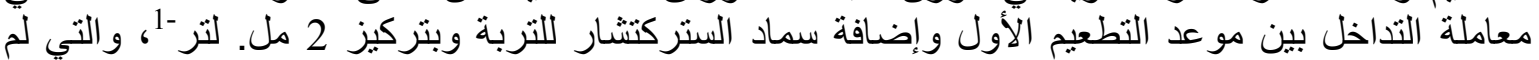

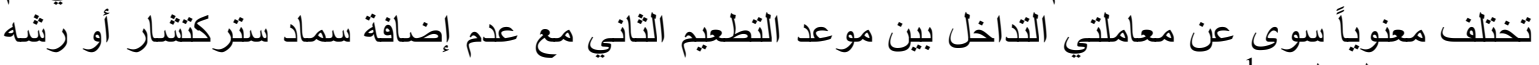

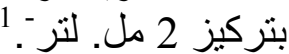
3. مساحة الأوراق (سم² / ورقة): يتضح من النتائج في الجدول (4) أن موعد التطعيم لم يؤثر معنوياً في

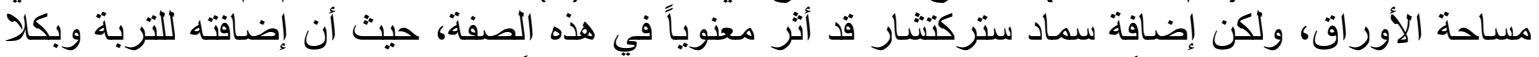

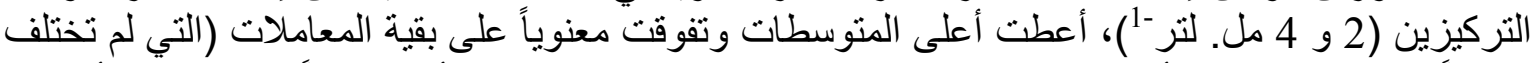

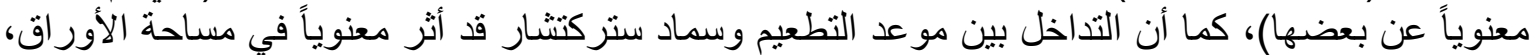

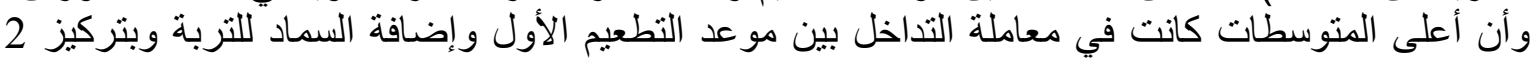


Mesopotamia J. of Agric.

Vol. (41) No. (2) 2013
ISSN:2224-9796(Online)

ISSN: 1815-316x (Print)

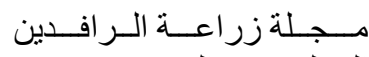

2013 المجلد (41) العدد (2)

مل. لتر -1 و التي تفوقت معنوياً على معاملات التداخل بين موعدي التطعيم الأول والثاني وعدم إضافة سماد

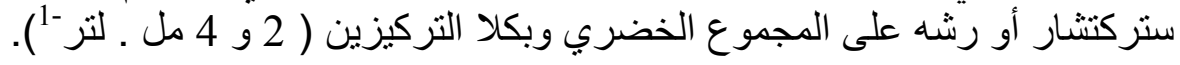

الجدول (2): تـأثثر موعد التطعيم ومستوى وطريقـة إضـافة سـماد ستركتشـار في الكلوروفيـل الكلي في

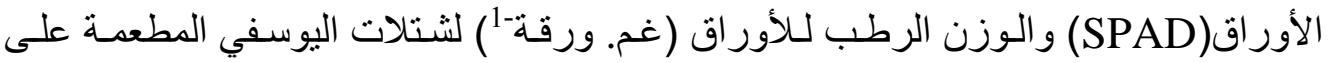

$$
\text { أصل النارنج البذري. }
$$

Table(2): Effect of budding date, level and method of structure fertilizer application on leaves total chlorophyll (SPAD) and leaves fresh weight (gm.leaf ${ }^{-1}$ ) of Tangarin transplants budded on seedlings of sour orange rootstock.

\begin{tabular}{|c|c|c|c|c|c|c|}
\hline \multicolumn{3}{|c|}{$\begin{array}{c}\text { الوزن الرطب للأوراق (غم) } \\
\text { Weight leaves fresh (gm) }\end{array}$} & \multicolumn{3}{|c|}{ الكلوروفيل ( SPAD ) } & \multirow{2}{*}{$\begin{array}{c}\text { الصفة } \\
\text { Parameterr } \\
\text { المعاملة/ مو عد } \\
\text { budding /Treatment } \\
\text { date } \\
\end{array}$} \\
\hline المنوسط & 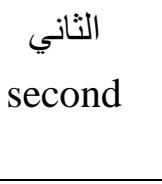 & 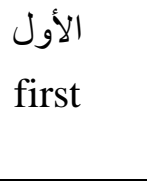 & المنوسط & 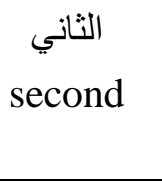 & 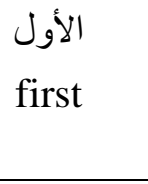 & \\
\hline $14.53 \mathrm{c}$ & $12.50 \mathrm{~d}$ & $16.57 \mathrm{a}-\mathrm{c}$ & $48.43 \mathrm{c}$ & $48.23 \mathrm{e}$ & $48.63 \mathrm{de}$ & الدقارنة (0) \\
\hline $15.23 \mathrm{bc}$ & $15.36 \mathrm{c}$ & $15.10 \mathrm{c}$ & $53.96 \mathrm{~b}$ & $55.00 \mathrm{~b}$ & $52.93 \mathrm{~b}-\mathrm{d}$ & 2 ml. foliar 2مل رش \\
\hline $15.40 \mathrm{bc}$ & $15.86 \mathrm{bc}$ & $14.93 c$ & $52.60 \mathrm{~b}$ & $50.36 \mathrm{c}-\mathrm{e}$ & $54.83 \mathrm{bc}$ & 4 ml. foliar 4 مل رش \\
\hline $16.48 \mathrm{~b}$ & $16.23 \mathrm{a}-\mathrm{c}$ & $16.73 \mathrm{a}-\mathrm{c}$ & $61.45 \mathrm{a}$ & $61.13 \mathrm{a}$ & $61.78 \mathrm{a}$ & 2 ml. soil 2 مل تربة \\
\hline \multirow[t]{2}{*}{$17.87 \mathrm{a}$} & $18.03 \mathrm{a}$ & $17.71 \mathrm{ab}$ & $63.01 \mathrm{a}$ & $64.20 \mathrm{a}$ & $61.83 \mathrm{a}$ & 4 ml. soilait 4 مل تربة \\
\hline & $15.60 \mathrm{a}$ & $16.21 \mathrm{a}$ & & $55.78 \mathrm{a}$ & $56.00 \mathrm{a}$ & المتوسط means \\
\hline
\end{tabular}

*المتوسطات المتبو عة بحروف مختلفة لكل عامل على إنفر اد وكذلك التداخل بينهما ولكل صفة تدل على وجود فروقات معنويـه

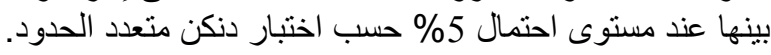

*Means of each factor alone and their interactions of each parameter followed with the same letters are not significantly different from each other's according to Duncan's multiple ranges test at $5 \%$ leve.

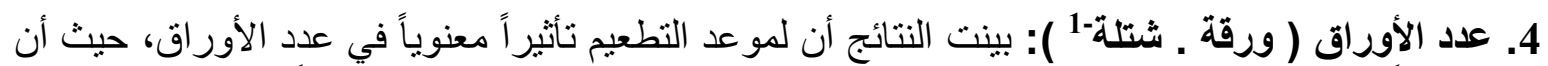

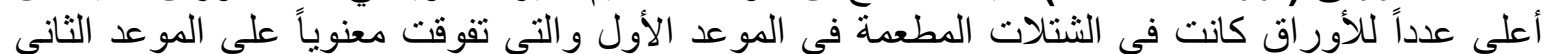

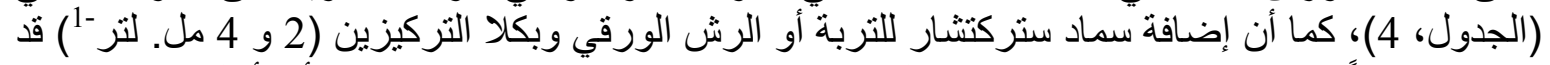

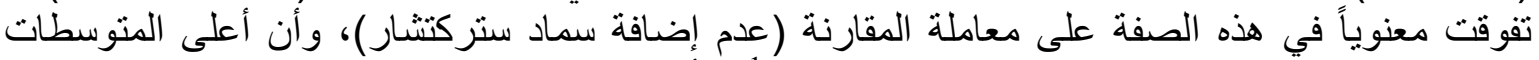

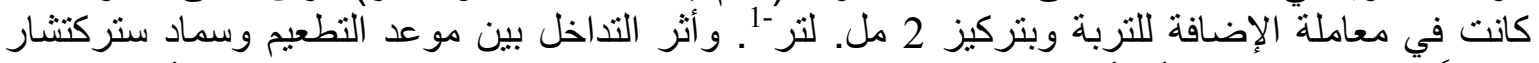

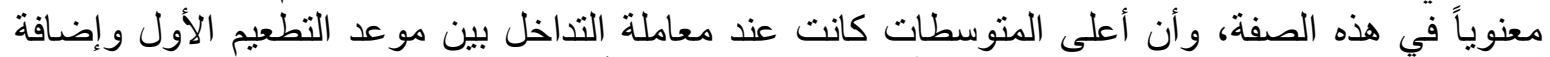

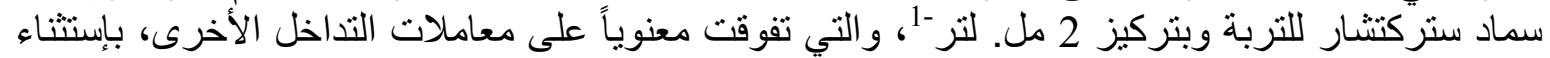

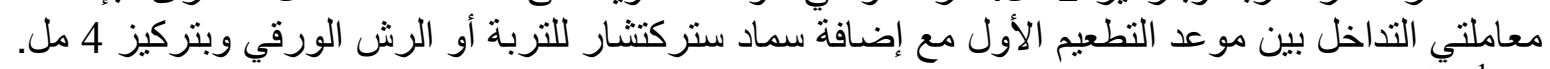
لتر -1 لكل منهما. 5. المساحة الورقية للشتلات (سم². شتلة-1): دلت النتائج أن أعلى مساحة ورقية للشتلات كانت في

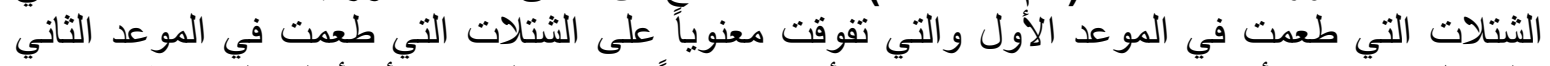

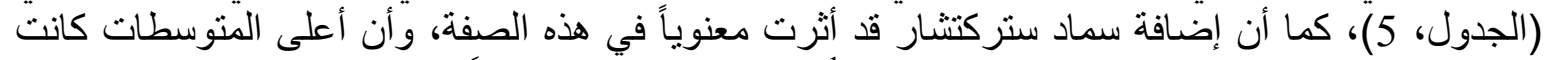

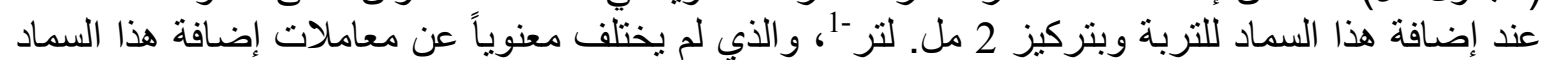

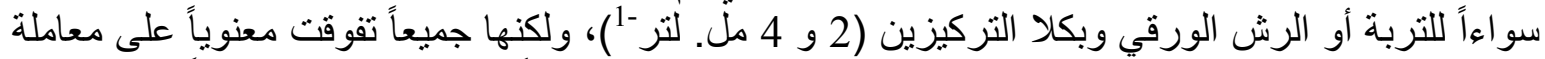

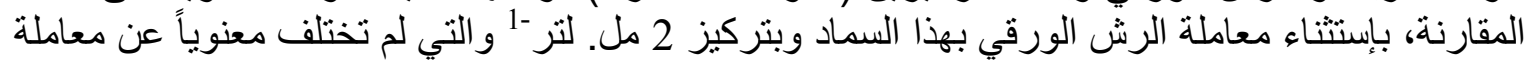


Mesopotamia J. of Agric.

Vol. (41) No. (2) 2013
ISSN:2224-9796(Online)

ISSN: 1815-316x (Print)

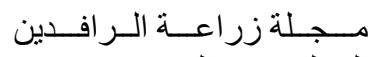

2013 المجلد (41) العدد (2)

المقارنة.

الجدول (3): تأثثر مو عد التطعيم ومستوى وطريقة إضافة سماد ستركتشار في الوزن الجاف للأوراق (غم) ونسبة المادة الجافة في الأوراق (\%) لثتانلات اليوسفي المطعمة على أصل النارنج البذري.

Table(3): Effect of budding date, level and method of structure fertilizer application on leaves dry weight $\left({\left.\mathrm{gm} . l e a f^{-1}\right)}\right.$ and leaf dry matter percentage $(\%)$ of Tangarin transplants budded on seedlings of sour orange rootstock.

\begin{tabular}{|c|c|c|c|c|c|c|}
\hline \multicolumn{3}{|c|}{$\begin{array}{c}\text { نسبة المادة الجافة في الأوراق ( \% ) } \\
\text { leaf dry matter } \\
\text { percentage(\%) }\end{array}$} & \multicolumn{3}{|c|}{ 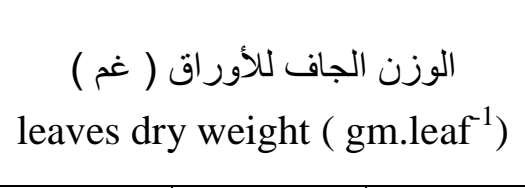 } & الصفة Parameter \\
\hline المتوسط & 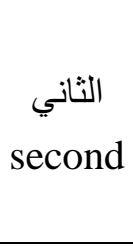 & 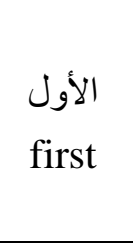 & المتوسط & 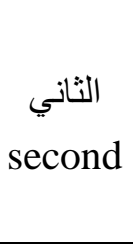 & 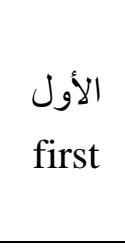 & $\begin{array}{c}\text { المعاملة/ مو عد التطعيم } \\
\text { budding /Treatment } \\
\text { date }\end{array}$ \\
\hline a 35.73 & a 37.01 & a 34.44 & b 5.17 & c 4.63 & a-c 5.71 & المقارنة (0) \\
\hline a35.07 & a 31.83 & a 38.32 & ab 5.34 & bc 4.88 & a-c 5.81 & $\begin{array}{l}2 \text { ml. 2مل رش } 2 \text { مoliar } \\
\text { fol }\end{array}$ \\
\hline a 33.86 & a 32.71 & $\mathrm{a} 35.02$ & b 5.24 & a-c 5.18 & a-c 5.30 & 4مل رش . 4 ml \\
\hline a 37.90 & a 36.94 & a 38.85 & a 6.20 & a-c 6.00 & a 6.50 & 2 ml. 2 مل نربة 2 \\
\hline a33.21 & a 33.17 & a 33.25 & ab 6.10 & a-c 6.00 & ab 6.21 & 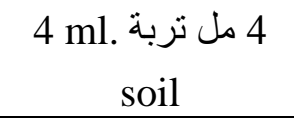 \\
\hline & a 34.33 & a 35.97 & & a 5.34 & a 5.90 & المتوسط means \\
\hline
\end{tabular}

*المتوسطات المنبو عة بحروف مختلفة لكل عامل على إنفر اد وكذلك التداخل بينهما ولكل صفة تدل على وجود فروقات معنويه

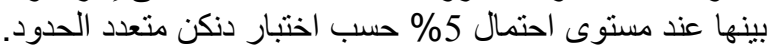

* Means of each factor alone and their interactions of each parameter followed with the same letters are not significantly different from each other's according to Duncan's multiple ranges test at $5 \%$ leve.

وكان للتداخل بين مو عد التطعيم وسماد ستركتشار نأثير اً معنوياً في المساحة الورقية للشتلات، حيث أن أعلى ألى

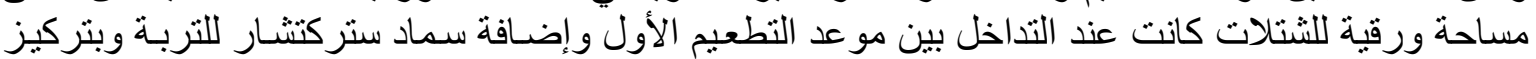

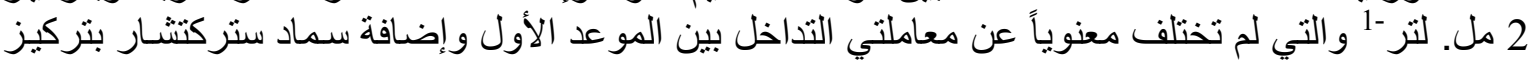

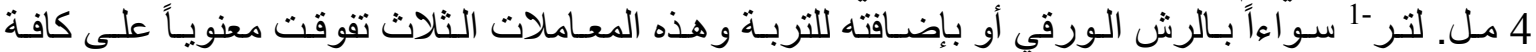

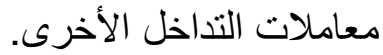
6. طول الساق الرئيس (سم): تؤكد النتائج الموضحة في الجدول (5)، أن موعد التطعيم الأول قد تفوق التوق

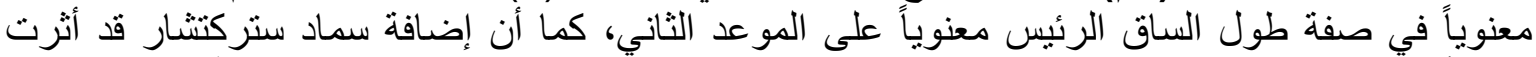

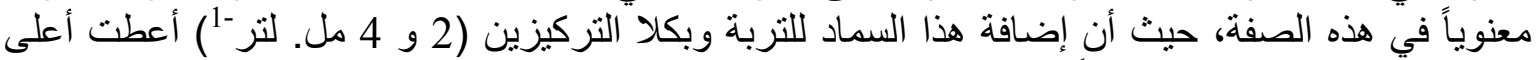

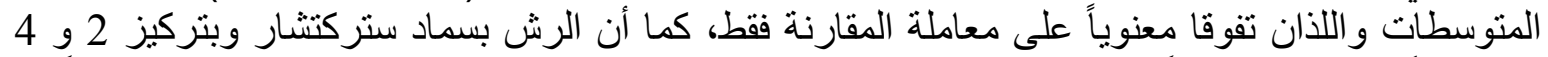

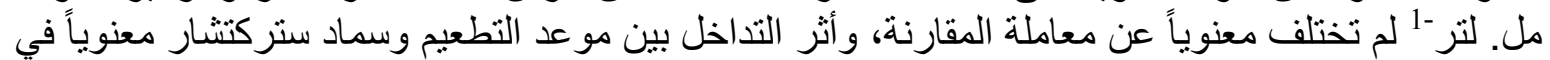

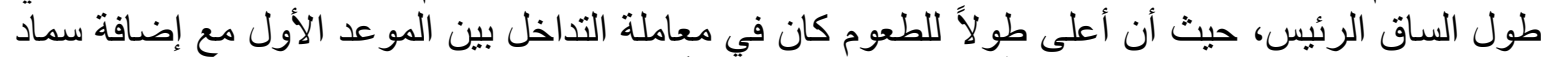

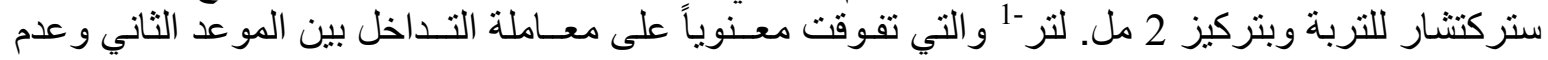


Mesopotamia J. of Agric.

Vol. (41) No. (2) 2013
ISSN:2224-9796(Online)

ISSN: 1815-316x (Print)

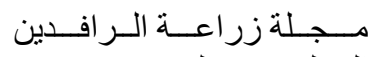

المجلد (41) العدد (2) 2013

إضافة السماد فقط.

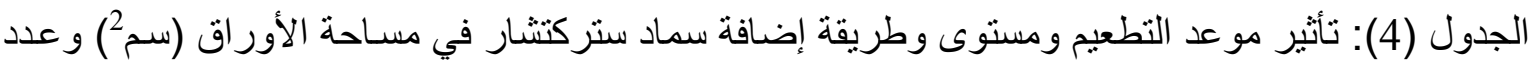

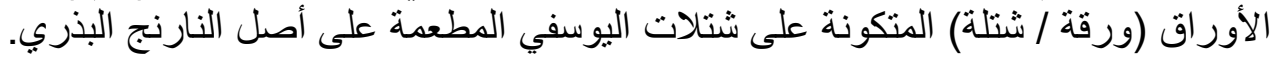

Table(4): Effect of budding date, level and method of structure fertilizer application on Leaf area $\left(\mathrm{cm}^{2}\right)$ and Leaves number ( leaf.transplant ${ }^{-1}$ ) of Tangarin transplants budded on seedlings of sour orange rootstock.

\begin{tabular}{|c|c|c|c|c|c|c|}
\hline \multicolumn{3}{|c|}{ 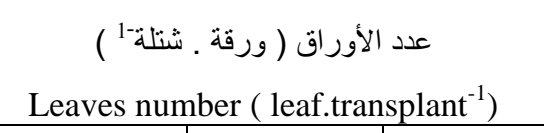 } & \multicolumn{3}{|c|}{$\begin{array}{c}\text { مساحة الورقة الواحدة ( سم² ) } \\
\text { Leaf area }\left(\mathrm{cm}^{2}\right)\end{array}$} & \multirow{2}{*}{$\begin{array}{c}\text { الصفة المعاملة/ مو عد التطعيم } \\
\text { parameter } \\
\text { budding /Treatment } \\
\text { date }\end{array}$} \\
\hline المتوسط & $\begin{array}{c}\text { الثاني } \\
\text { second }\end{array}$ & 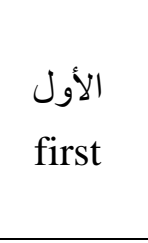 & المتوسط & 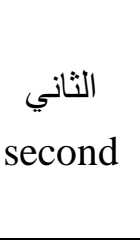 & 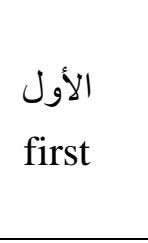 & \\
\hline b 42.83 & c 25.33 & bc 60.33 & b 29.88 & e 29.00 & c-e30.76 & المقارنة (0) \\
\hline a 82.17 & bc 70.00 & b 94.33 & b 31.59 & de 30.18 & b-e33.00 & 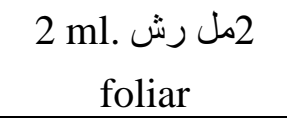 \\
\hline a 107.00 & bc 60.00 & a 154.00 & b 32.36 & b-e31.73 & b-e 33.00 & $\begin{array}{c}4 \text { ml. 4مل رش } 4 \text { foliar } \\
\end{array}$ \\
\hline a 107.33 & bc 66.00 & a 148.67 & a 37.89 & a-d 35.60 & a 40.18 & $\begin{array}{l}2 \text { ml. } 2 \text { مل تربة } 2 \text { soil } \\
\text { sil }\end{array}$ \\
\hline a 99.67 & bc 53.67 & a 145.67 & a 37.50 & a-c 37.00 & ab 38.00 & $\begin{array}{l}4 \text { ml. مل تربة } 4 \text { soil } \\
\text { sil }\end{array}$ \\
\hline & b 55.00 & a 120.60 & & a 32.70 & a 34.98 & means المتوسط \\
\hline
\end{tabular}

*المتوسطات المتنو عة بحروف مختلفة لكل عامل على إنفر اد وكذلك التداخل بينهما ولكل صفة تدل على وجود فروقات معنويه

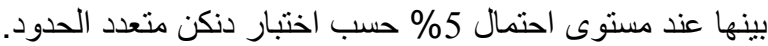

* Means of each factor alone and their interactions of each parameter followed with the same letters are not significantly different from each other's according to Duncan's multiple ranges test at $5 \%$ leve.

7. قطر الساق الرئيس (ملم): لقد أثر موعد التطعيم وإضافة سماد ستركتشار والتداخل بينهما معنوياً في

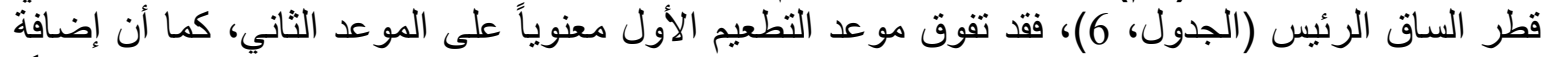

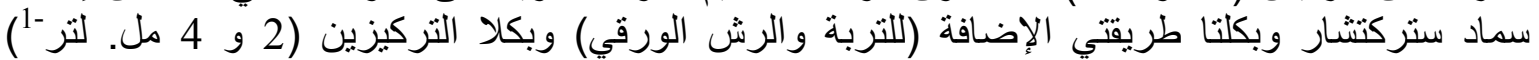

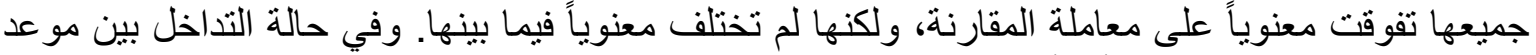

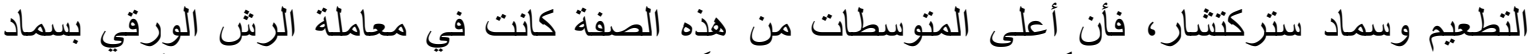

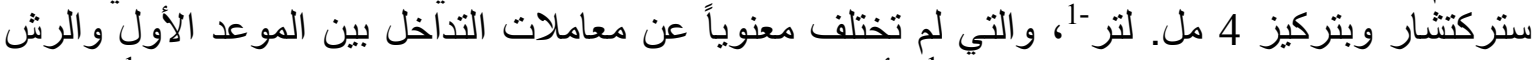

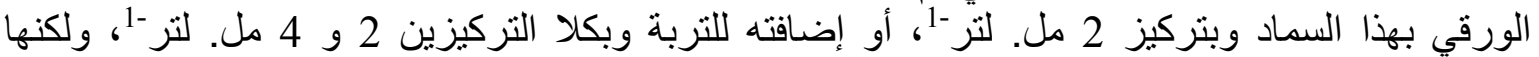
تفوقت معنوياً على معاملات التداخل التخل الأخرى.

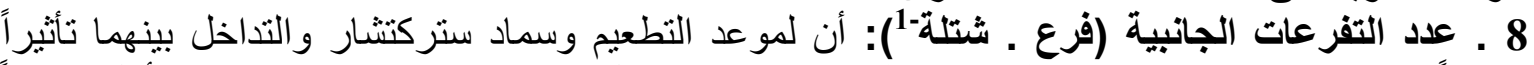

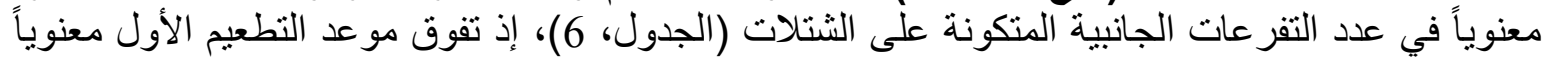

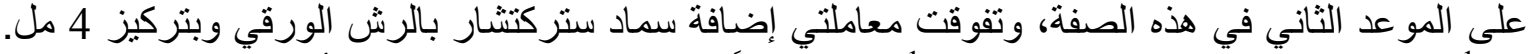

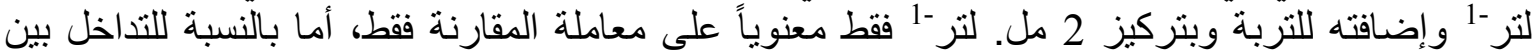

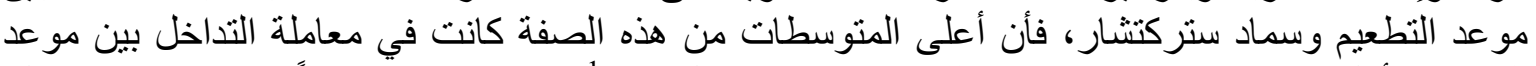

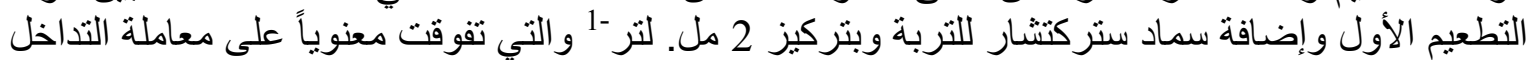


Mesopotamia J. of Agric.

Vol. (41) No. (2) 2013
ISSN:2224-9796(Online)

ISSN: 1815-316x (Print)

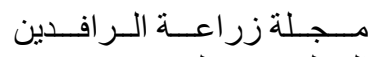

2013 المجلد (41) العدد (2)

بين مو عد التطعيم الثاني و عدم إضافة سماد ستركتشار فقط.

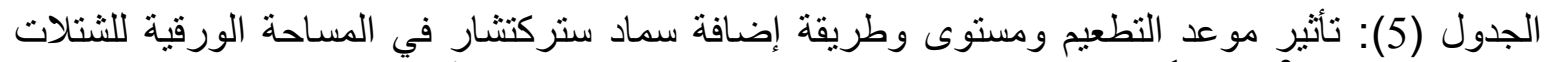

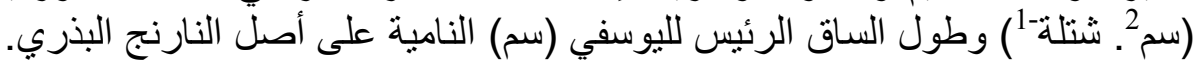

Table(5): Effect of budding date, level and method of structure fertilizer application on Transplants leaves area (cm2.transplant-1) and Main stem length (cm)of Tangarin transplants budded on seedlings of sour orange rootstock.

\begin{tabular}{|c|c|c|c|c|c|c|}
\hline \multicolumn{3}{|c|}{$\begin{array}{c}\text { طول الساق الرئيس ( سم ) } \\
\text { Main stem length (cm) }\end{array}$} & \multicolumn{3}{|c|}{$\begin{array}{c}\text { المساحة الورقية للشتلات ( سم2 ـ شتلة-1 ) } \\
\text { Transplant leaves area } \\
\text { (cm2.transplant-1) }\end{array}$} & $\begin{array}{c}\text { الصفة } \\
\text { Parameter }\end{array}$ \\
\hline المنوسط & 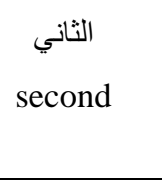 & $\begin{array}{ll}-1 \\
\text { first }\end{array}$ & المتوسط & $\begin{array}{c}\text { الثاني } \\
\text { second }\end{array}$ & الم الاول & $\begin{array}{c}\text { budding / Treatment } \\
\text { date }\end{array}$ \\
\hline $48.12 \mathrm{~b}$ & $35.88 \mathrm{c}$ & $60.37 \mathrm{a}-\mathrm{c}$ & $1294.3 \mathrm{~b}$ & $732.8 \mathrm{c}$ & $1855.9 \mathrm{bc}$ & المقارنة (0) \\
\hline $65.70 \mathrm{ab}$ & $66.38 \mathrm{a}-\mathrm{c}$ & $65.03 \mathrm{a}-\mathrm{c}$ & 2614.3ab & $2137.2 \mathrm{bc}$ & $3091.5 b$ & $\begin{array}{l}2 \mathrm{ml} \text { 2مل رش } 2 \text { foliar }\end{array}$ \\
\hline 67.29ab & $50.69 \mathrm{bc}$ & $83.89 a$ & $3508.8 \mathrm{a}$ & $1889.4 \mathrm{bc}$ & $5128.2 \mathrm{a}$ & $\begin{array}{c}4 \text { ml. } 4 \text { رل رش } \\
\text { foliar }\end{array}$ \\
\hline $73.94 \mathrm{a}$ & $61.88 \mathrm{a}-\mathrm{c}$ & $86.00 \mathrm{a}$ & $4149.3 \mathrm{a}$ & $2345.5 b c$ & $5953.2 \mathrm{a}$ & 2 ml. 2 مل تربة \\
\hline $70.83 \mathrm{a}$ & $61.67 \mathrm{a}-\mathrm{c}$ & $80.00 \mathrm{ab}$ & $3844.7 \mathrm{a}$ & $1956.9 \mathrm{bc}$ & $5732.5 \mathrm{a}$ & 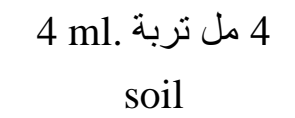 \\
\hline & $55.30 \mathrm{~b}$ & $75.05 \mathrm{a}$ & & $1812.3 \mathrm{~b}$ & $4352.2 \mathrm{a}$ & means المتوسط \\
\hline
\end{tabular}

*المتوسطات المتبو عة بحروف مختلفة لكل عامل على إنفر اد وكذلك التداخل بينهما ولكل صفة تدل على وجود فرود فروقات معنويـه بينها عند مستوى احتمال 5\% \% مسب اختبار دنكن متعدد الحدود.

*Means of each factor alone and their interactions of each parameter followed with the same letters are not significantly different from each other's according to Duncan's multiple ranges test at $5 \%$ leve.

إن تفوق موعد التطعيم الأول على الموعد الثاني في بعض الصفات (عدد الأوراق والمساحة الورقية

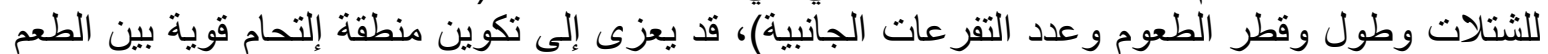

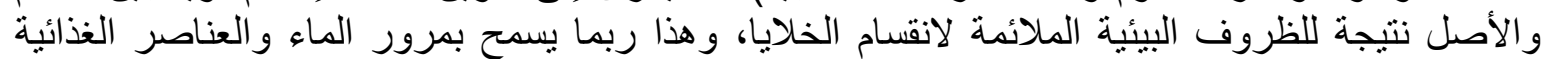

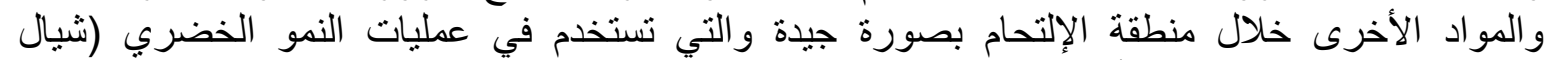
وآخرون، 2010)، في حين أن الزيادة في صفات النمو الخضري نتيجة لإضافة سماد ستركتشار وكذللك

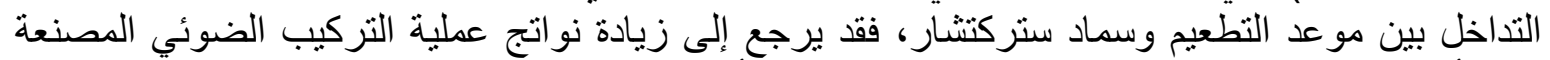

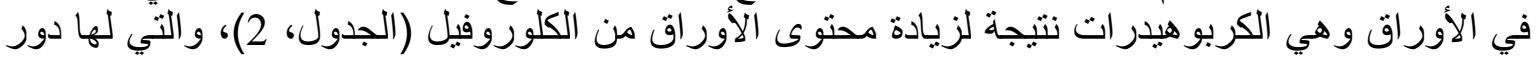

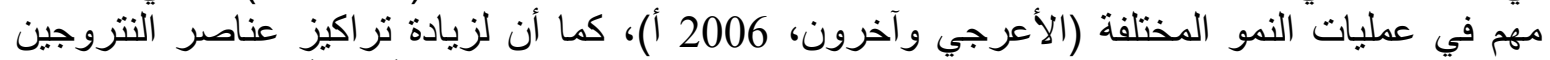

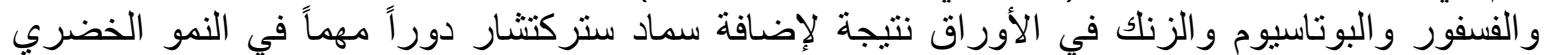

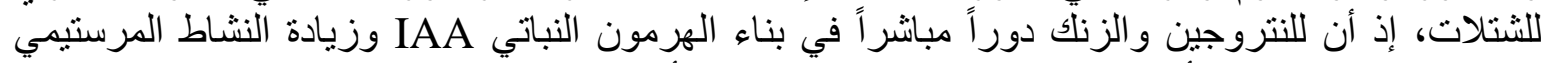

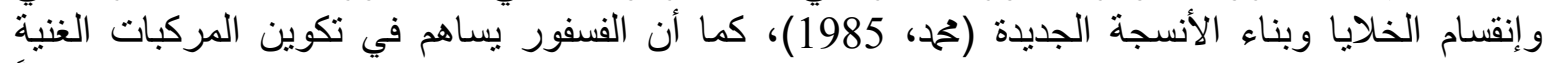

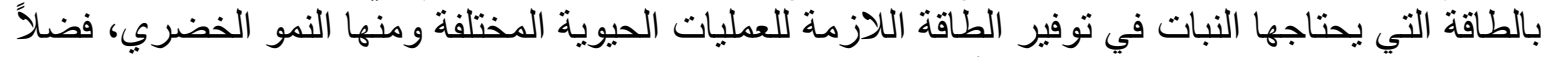

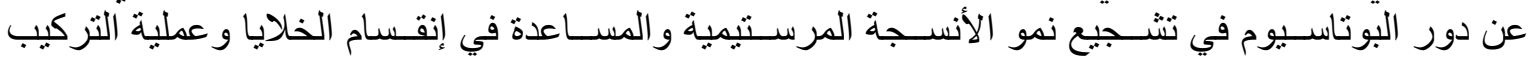


Mesopotamia J. of Agric.

Vol. (41) No. (2) 2013
ISSN:2224-9796(Online)

ISSN: 1815-316x (Print)

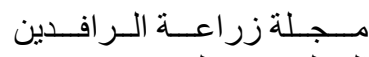

2013 المجلد (41) العدد (2)

الضوئي وانتقال المواد الناتجة من هذه العملية وتنشيط العديد من الإنزيمات (جندية، 2003)،

الجدول (6): تأثير مو عد التطعيم ومستوى وطريقة إضـافة سماد ستركتشـار في قطر السـاق الرئيس (ملم)

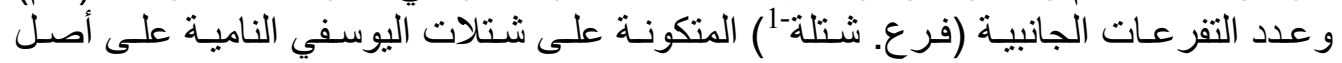

النارنج البذري.

Table(6): Effect of budding date, level and method of structure fertilizer application on Main stem diameter ( $\mathrm{mm}$ ) and Lateral branches number ( branch.transplant ${ }^{-1}$ ) of Tangarin transplants budded on seedlings of sour orange rootstock.

\begin{tabular}{|c|c|c|c|c|c|c|}
\hline \multicolumn{3}{|c|}{$\begin{array}{c}\text { عدد التفر عات الجانبية ( فرع. شتلة-1' ) } \\
\text { Lateral branches number } \\
\text { (branch.transplant }{ }^{-1} \text { ) }\end{array}$} & \multicolumn{3}{|c|}{$\begin{array}{c}\text { قطر الساق الرئيس (ملم) } \\
\text { Main stem diameter (ml) }\end{array}$} & $\begin{array}{c}\text { الصفة } \\
\text { Parameter }\end{array}$ \\
\hline المنوسط & $\begin{array}{c}\text { الثاني } \\
\text { second }\end{array}$ & 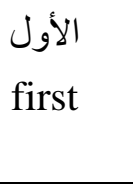 & المتوسط & $\begin{array}{c}\text { الثاني } \\
\text { second }\end{array}$ & 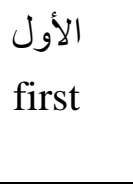 & $\begin{array}{c}\text { التطعيم } \\
\text { budding /Treatment } \\
\text { date } \\
\end{array}$ \\
\hline $3.55 \mathrm{~b}$ & $3.22 \mathrm{~b}$ & $3.88 \mathrm{ab}$ & $4.44 \mathrm{~b}$ & $3.56 \mathrm{e}$ & $5.31 \mathrm{~d}$ & المقارنة (0) \\
\hline $5.66 \mathrm{ab}$ & $4.55 \mathrm{ab}$ & $6.77 \mathrm{ab}$ & $6.75 \mathrm{a}$ & $6.45 b-d$ & $7.05 \mathrm{a}-\mathrm{c}$ & 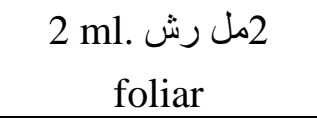 \\
\hline $6.27 \mathrm{a}$ & $6.10 \mathrm{ab}$ & 6.44ab & $6.66 \mathrm{a}$ & $5.05 \mathrm{~d}$ & $8.28 \mathrm{a}$ & $\begin{array}{l}4 \text { ml. 4مل رش } 4 \text { foliar } \\
\text { for }\end{array}$ \\
\hline $5.99 \mathrm{a}$ & $4.88 \mathrm{ab}$ & $7.11 \mathrm{a}$ & $6.86 \mathrm{a}$ & $6.00 \mathrm{~cd}$ & $7.73 \mathrm{ab}$ & 2 ml. soillaبة مل تربة \\
\hline $5.16 \mathrm{ab}$ & $3.77 \mathrm{ab}$ & $6.55 \mathrm{ab}$ & $6.50 \mathrm{a}$ & $5.36 \mathrm{~d}$ & $7.63 \mathrm{ab}$ & 4 ml. soila مل تربة \\
\hline & $4.50 \mathrm{~b}$ & $6.15 \mathrm{a}$ & & $5.28 \mathrm{~b}$ & $7.20 \mathrm{a}$ & means المتوسط \\
\hline
\end{tabular}

*المتوسطات المتبو عة بحروف مختلفة لكل عامل على إنفر اد وكذلك التداخل بينهما ولكل صفة تدل على وجلى وجود فروقات معنويهـ بينها عند

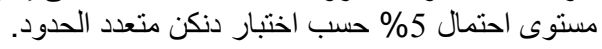

* Means of each factor alone and their interactions of each parameter followed with the same letters are not significantly different from each other's according to Duncan's multiple ranges test at 5\% leve.

وللزنك دور في توسع واستطالة الخلايا النباتية وتكوين سكر الكلوكوز وينشط عدد من الأنزيمات منها وكإن Carbonic anhydrase

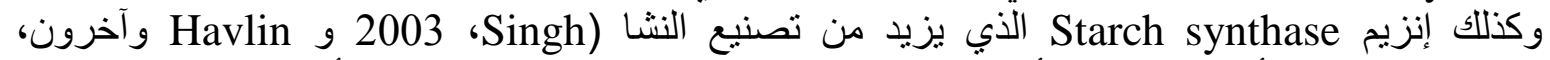

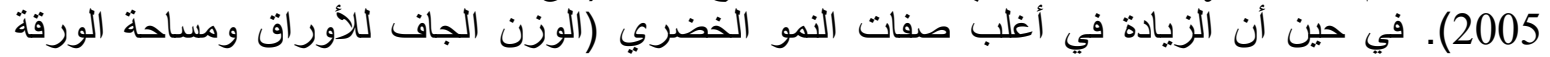

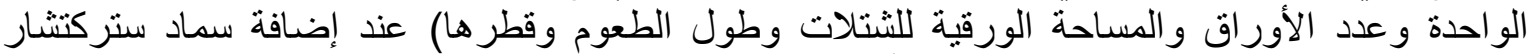

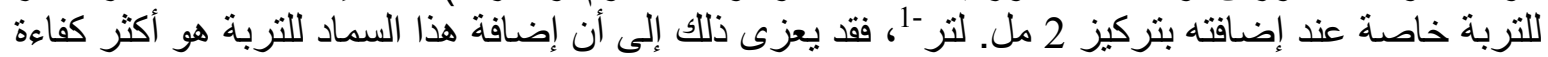

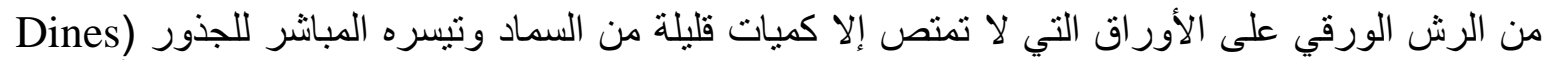

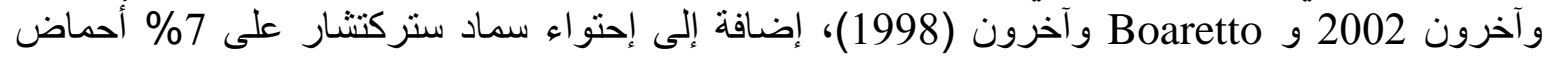

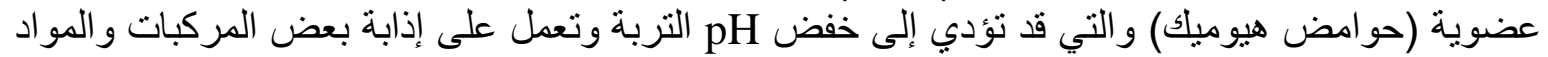

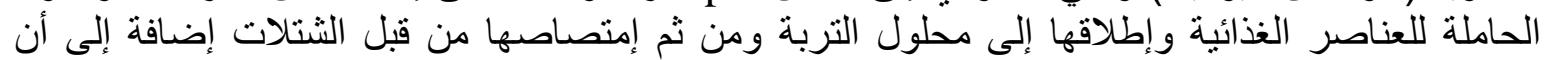

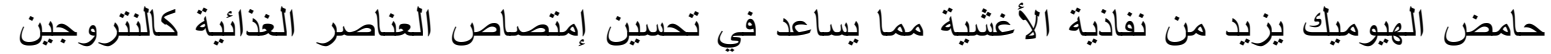

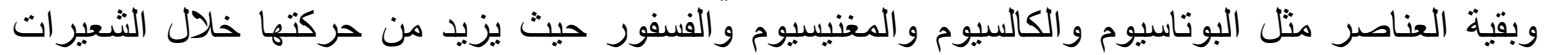

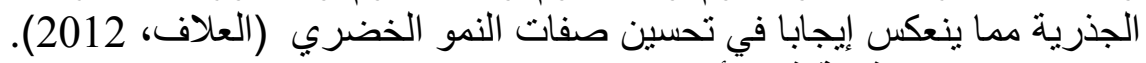

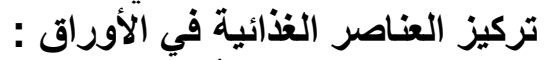
1. النتروجين (\%): أكدت النتائج الموضحة في في الجدول فئن (7)، أن موعد التطعيم لم يؤثر معنوياً في تركيز

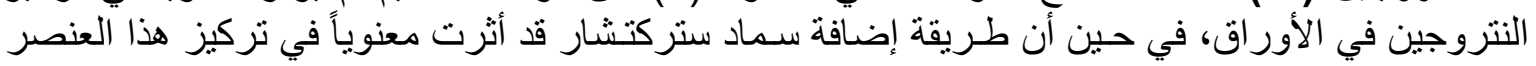


Mesopotamia J. of Agric.

Vol. (41) No. (2) 2013
ISSN:2224-9796(Online)

ISSN: 1815-316x (Print)

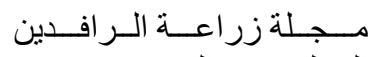

2013 المجلد (41) العدد (2)

في الأوراق، فقد أعطت معاملة الإضافة للتربة وبتركيز 2 مل. لتر -1 أعلى المتوسطات والتي تفوقت معنوياً

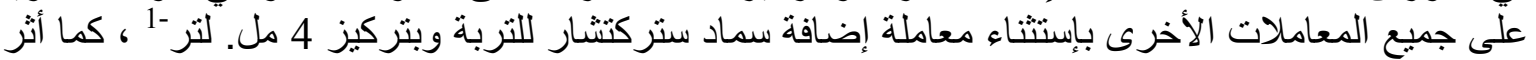

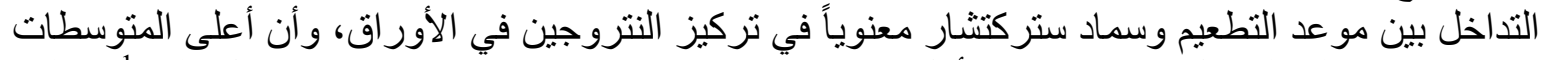

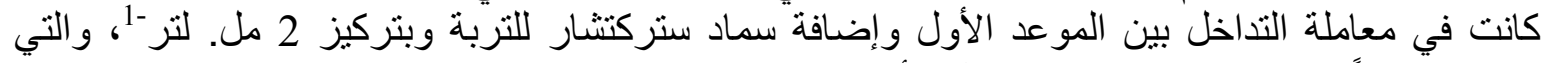

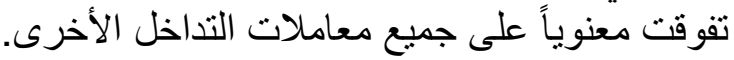

الجدول (7): تأثير موعد التطعيم ومستوى وطريقة إضافة سماد ستركتشار في نركيز النتروجين و الفسفور(\%) في أوراق شتنات اليوسفي النامية على أصل النارنج البذري.

Table(7): Effect of budding date, level and method of structure fertilizer application on $\mathrm{N}(\%)$ and $\mathrm{P}(\%)$ of Tangarin transplants budded on seedlings of sour orange rootstock .

\begin{tabular}{|c|c|c|c|c|c|c|}
\hline \multicolumn{3}{|c|}{ P(\%) الفسفور P(\% } & \multicolumn{3}{|c|}{ النتروجين N(\% } & parameter الصفة \\
\hline $\begin{array}{l}\text { المتوسط } \\
\text { Means }\end{array}$ & $\begin{array}{c}\text { الثاني } \\
\text { second }\end{array}$ & $\begin{array}{l}\text { الأول } \\
\text { first }\end{array}$ & $\begin{array}{l}\text { المتوسط } \\
\text { means }\end{array}$ & $\begin{array}{r}\text { الثاني } \\
\text { second }\end{array}$ & $\begin{array}{l}\text { الأول } \\
\text { first }\end{array}$ & $\begin{array}{c}\text { المعاملة/ مو عد التطعيم } \\
\text { budding / Treatment } \\
\text { date }\end{array}$ \\
\hline $0.363 \mathrm{a}$ & $0.416 \mathrm{a}$ & $0.310 \mathrm{ab}$ & $0.71 \mathrm{~d}$ & $0.81 \mathrm{ef}$ & $0.61 \mathrm{f}$ & المقارنة (0) \\
\hline $0.406 \mathrm{a}$ & $0.403 \mathrm{a}$ & $0.410 \mathrm{a}$ & $1.10 \mathrm{c}$ & $1.22 \mathrm{~cd}$ & $0.97 \mathrm{de}$ & 2 ml. foliar 2مل رش \\
\hline $0.370 \mathrm{a}$ & $0.440 \mathrm{a}$ & $0.300 \mathrm{ab}$ & $1.38 \mathrm{~b}$ & $1.30 \mathrm{c}$ & $1.466 \mathrm{bc}$ & 4 ml. foliar 4مل رش \\
\hline $0.220 \mathrm{~b}$ & $0.223 \mathrm{~b}$ & $0.216 \mathrm{~b}$ & $1.62 \mathrm{a}$ & $1.22 \mathrm{~cd}$ & $2.03 \mathrm{a}$ & 2 ml. soil مل ترببة \\
\hline $0.181 \mathrm{~b}$ & $0.180 \mathrm{~b}$ & $0.183 \mathrm{~b}$ & $1.46 \mathrm{ab}$ & $1.59 \mathrm{~b}$ & $1.34 \mathrm{bc}$ & 4 ml. soil مل تربة \\
\hline & $0.332 \mathrm{a}$ & $0.284 \mathrm{a}$ & & $1.23 \mathrm{a}$ & $1.28 \mathrm{a}$ & means المتوسط \\
\hline
\end{tabular}

*المتوسطات المتبو عة بحروف مختلفة لكل عامل على إنفر اد وكذلك التداخل بينهما ولكل صفة تدل على وجود فروقات معنويهـ

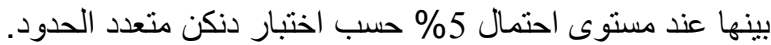

*Means of each factor alone and their interactions of each parameter followed with the same letters are not significantly different from each other's according to Duncan's multiple ranges test at $5 \%$ leve.

2. الفسفور (\%): توضح النتائج المبينة في الجدول (7)، أن موعد التطعيم لم يؤثر معنوياً في تركيز

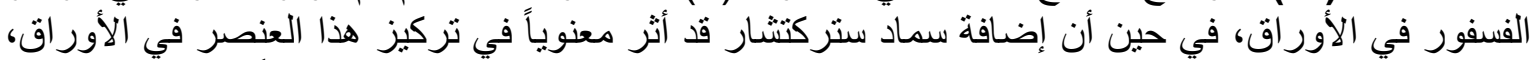

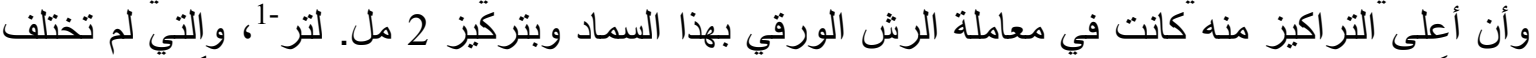

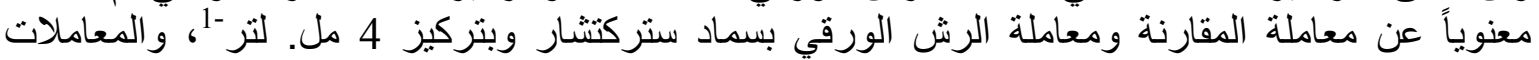

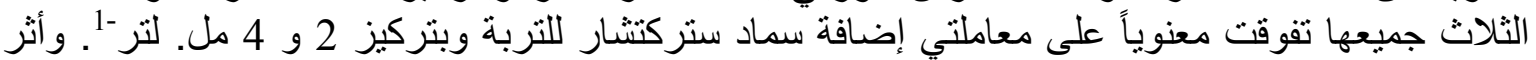

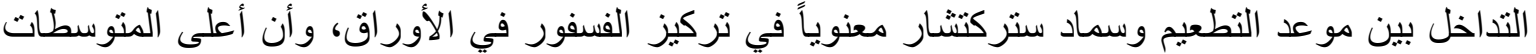

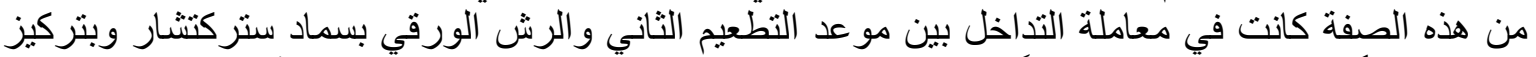

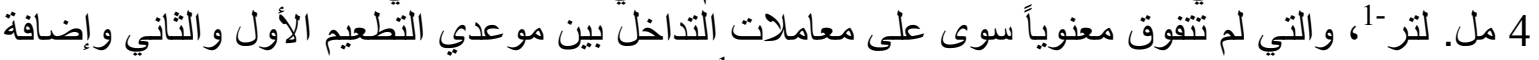

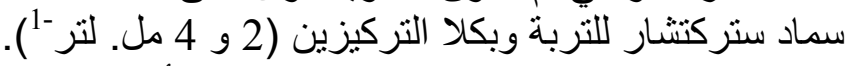

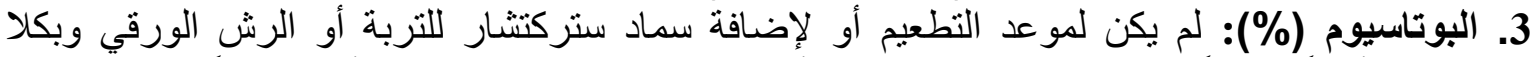

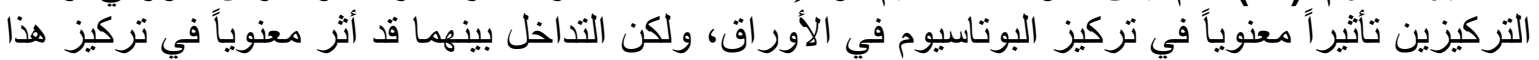

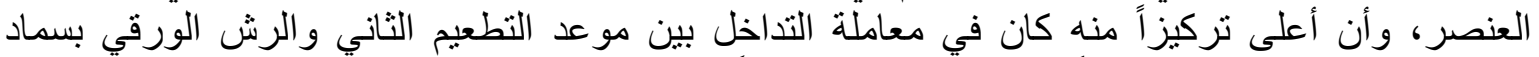

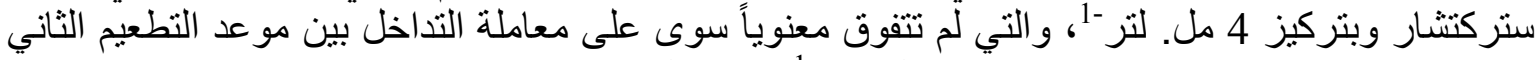

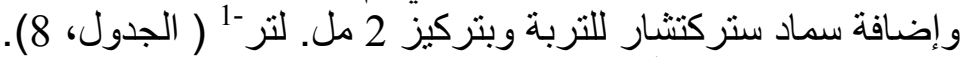

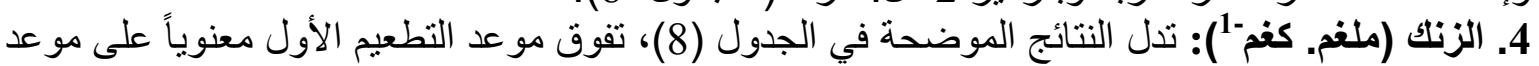

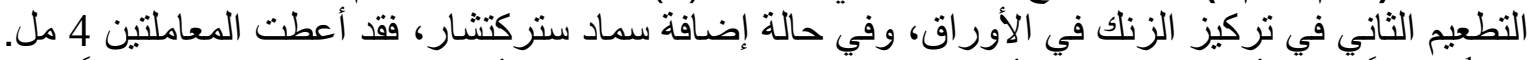

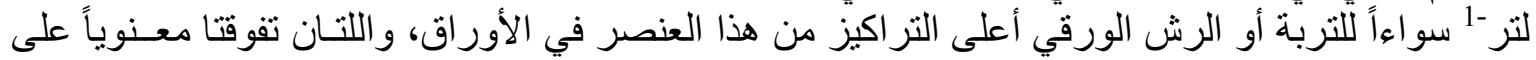


Mesopotamia J. of Agric.

Vol. (41) No. (2) 2013
ISSN:2224-9796(Online)

ISSN: 1815-316x (Print)

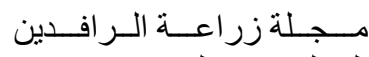

2013 المجلد (41) العدد (2)

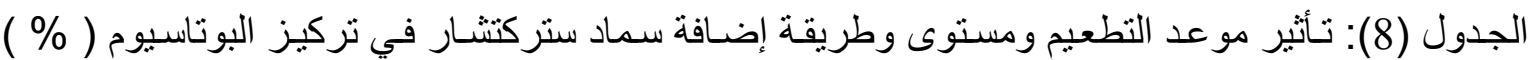

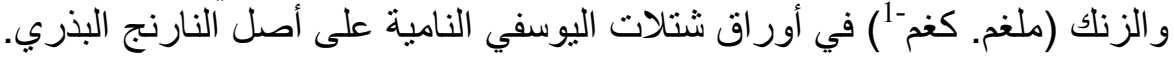

Table(8): Effect of budding date, level and method of structure fertilizer application on $\mathrm{K}(\%)$ and $\mathrm{Zn}\left(\mathrm{mg} \cdot \mathrm{kg}^{-1}\right)$ of Tangarin transplants budded on seedlings of sour orange rootstock.

\begin{tabular}{|c|c|c|c|c|c|c|}
\hline \multicolumn{3}{|c|}{ Zn (mg.kg $\left.{ }^{-1}\right)$ الزنك ( ملغم . كغم ) } & \multicolumn{3}{|c|}{ البوتاسيوم ( \% م ) } & \multirow{2}{*}{$\begin{array}{c}\text { الصففة Parameter الصعلة/ مو عد التطعيم } \\
\text { budding / Treatment } \\
\text { date }\end{array}$} \\
\hline المتوسط & $\begin{array}{c}\text { الثاني } \\
\text { second }\end{array}$ & $\begin{array}{l}\text { الأول } \\
\text { first }\end{array}$ & المنوسط & $\begin{array}{c}\text { الثاني } \\
\text { second }\end{array}$ & $\begin{array}{l}\text { الأول } \\
\text { first }\end{array}$ & \\
\hline $42.80 \mathrm{c}$ & $31.66 \mathrm{c}$ & $53.95 \mathrm{ab}$ & $1.23 \mathrm{a}$ & $1.16 \mathrm{ab}$ & $1.30 \mathrm{a}$ & المقارنة (0) \\
\hline $49.97 \mathrm{ab}$ & $46.62 b$ & $53.33 \mathrm{ab}$ & $1.25 \mathrm{a}$ & $1.18 \mathrm{ab}$ & $1.31 \mathrm{a}$ & $\begin{array}{c}2 \text { ml. } 2 \text { مل رش } 2 \text { foliar } \\
\end{array}$ \\
\hline $53.12 \mathrm{a}$ & $53.95 \mathrm{ab}$ & $52.29 \mathrm{ab}$ & $1.26 \mathrm{a}$ & $1.31 \mathrm{a}$ & $1.21 \mathrm{ab}$ & 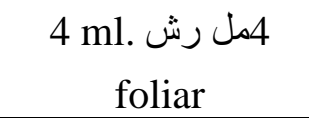 \\
\hline $47.20 \mathrm{bc}$ & $38.41 \mathrm{c}$ & $56.00 \mathrm{a}$ & $1.183 \mathrm{a}$ & $1.15 \mathrm{~b}$ & $1.21 \mathrm{ab}$ & 2 ml. soil 2 مل تربة \\
\hline $53.24 \mathrm{a}$ & $54.66 \mathrm{ab}$ & $51.82 \mathrm{ab}$ & $1.22 \mathrm{a}$ & $1.21 \mathrm{ab}$ & $1.23 \mathrm{ab}$ & 4 ml. soil مل ترببة \\
\hline & $45.06 \mathrm{~b}$ & $53.47 \mathrm{a}$ & & $1.20 \mathrm{a}$ & $1.25 \mathrm{a}$ & المتوسط means \\
\hline
\end{tabular}

*المتو سطات المتبو عة بحروف مختلفة لكل عامل على إنفر اد وكذلك التداخل بينهما ولكل صفة تدل على وجود فروقات معنويـاه بينها عند مستوى احتمال 5\% \% مسب اختبار دنكن متعدد الحدود.

*Means of each factor alone and their interactions of each parameter followed with the same letters are not significantly different from each other's according to Duncan's multiple ranges test at $5 \%$ leve.

معاملتي المقارنة و إضافة سماد ستركتشار للتربة وبتركيز 2 مل. لتر -1. وعند التداخل بين موعد التماد التطعيم

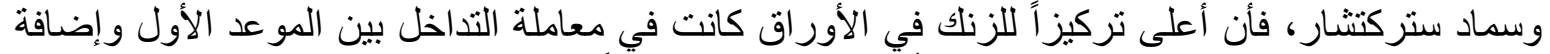

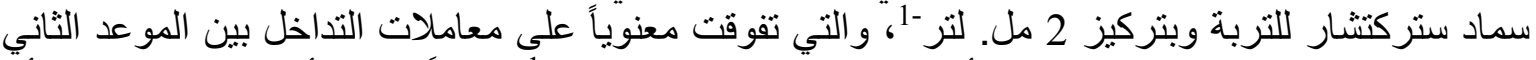

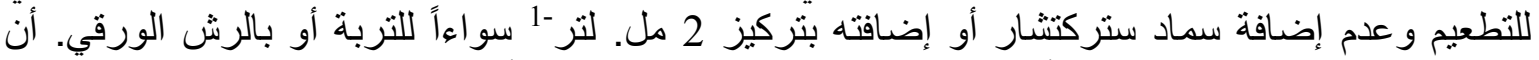

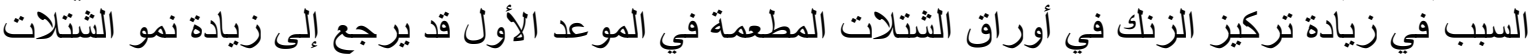

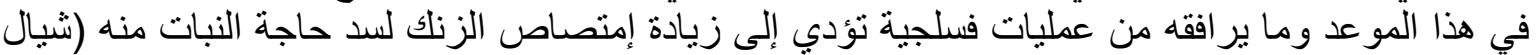

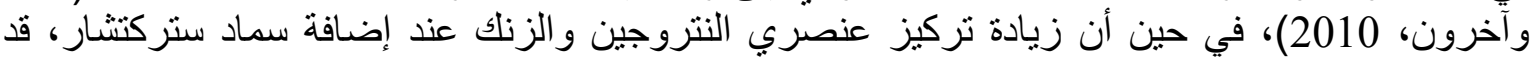

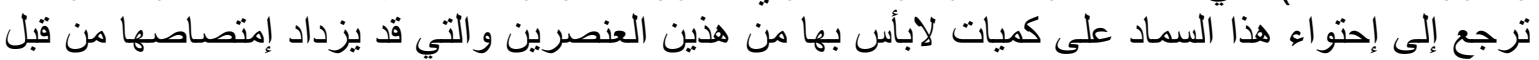

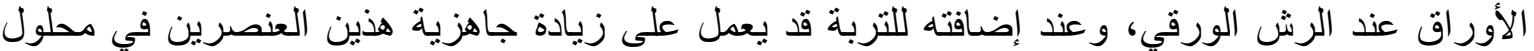

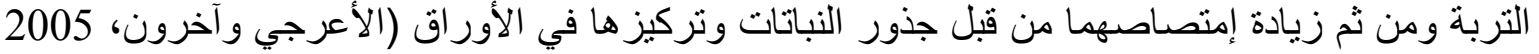

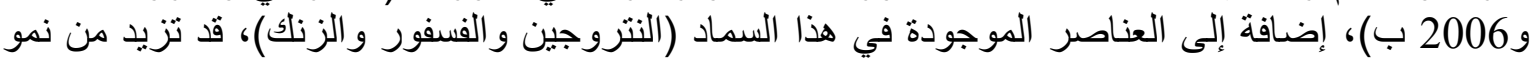

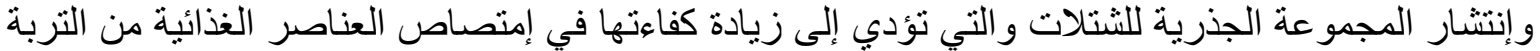

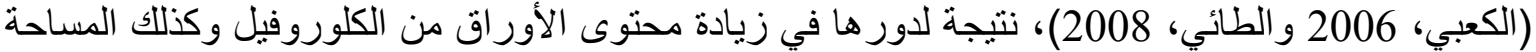

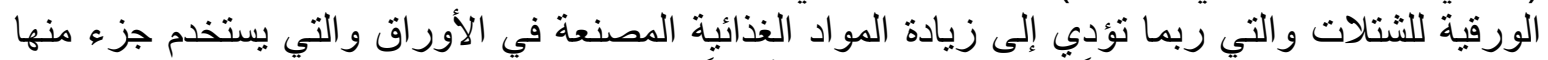

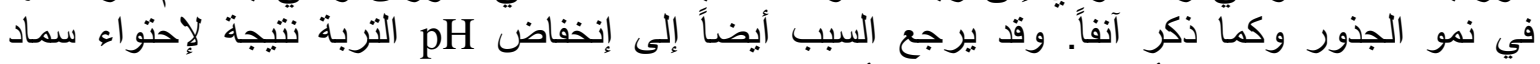

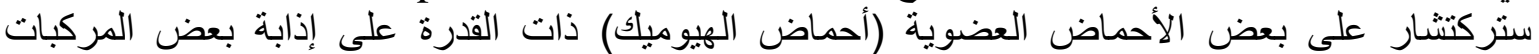

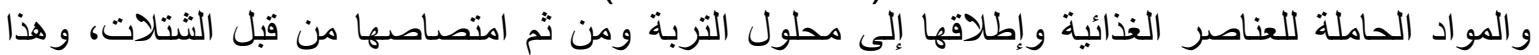

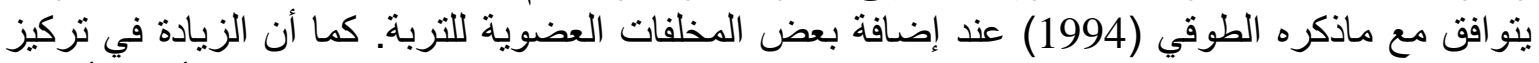

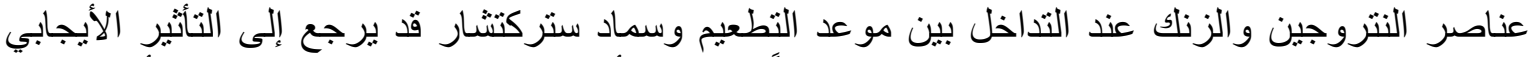

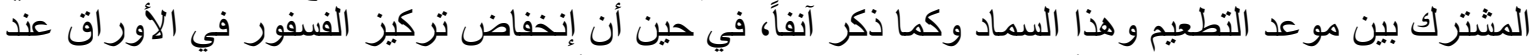

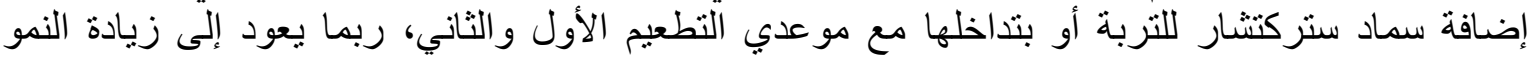


Mesopotamia J. of Agric.

Vol. (41) No. (2) 2013
ISSN:2224-9796(Online)

ISSN: 1815-316x (Print)

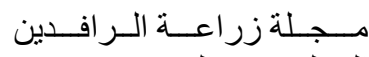

2013 المجلد (41) العدد (2)

الخضري للشتلات في هذه المعاملات (الجداول 2 - 6)، و عدم كفاية الفسفور الممتص من التربة لتلبية

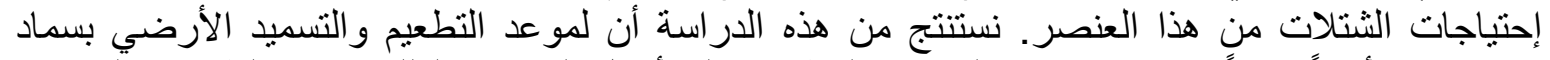

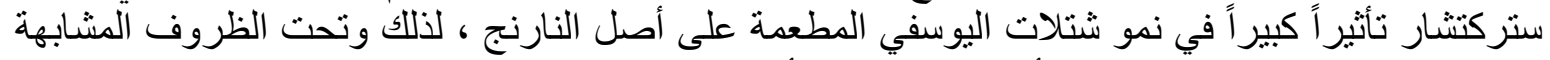

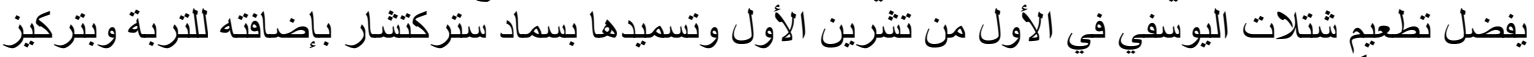

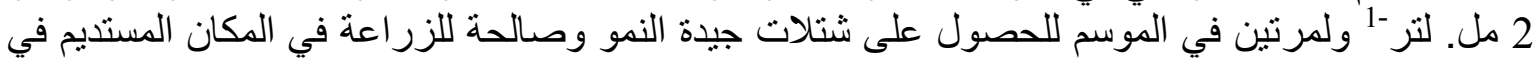

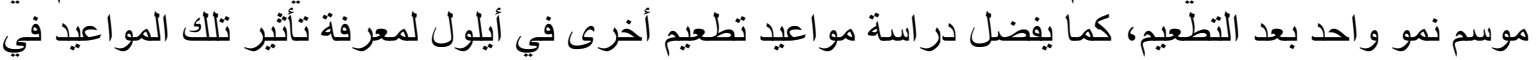
نمو هذه الثنتلات.

\title{
STUDTYING THE RESPONSE OF TANGARIN SCIONS GROWN ON SOUR ORANG ROTSTOCK FOR BUDDING DATE, APPLICATION METHOD AND LEVELS OF STRUCTUR ACTA AGRO COMPOUND FERTILIZER
}

\author{
Jassim M.Al-A'areji Ayad H. Al-Allaf Ayad T. Shayal Alalam
}

Hort.\& Landscape Design Dept. College of Agric.\& Foresty, Mosul Univ. Iraq.

\begin{abstract}
This study were conducted at lath house / Hort . \& Landscape Design/ Coll. of Agriculture and Forestry during 2010 and 2011 growing seasons on Tangarin transplants which were budded on sour orange rootstock to know the effect of autumn budding date (1/10/2010 and 15/10/2010) and foliar or soil application of structure fertilizer at two levels for each method $\left(2\right.$ and $\left.4 \mathrm{ml} . \mathrm{L}^{-1}\right)$. The application of the fertilizer was done twice a season, with 20 days intervals, the first was done when the scion length was $45 \mathrm{~cm}$ (at 15/4/2011). Results indicated that the first date of budding was dominated over the second date of budding in the leaves Zn level, transplants leave number, transplants leaves area, main stem length and diameter and lateral branches number. For the effect of method application and levels of structure fertilizer, results showed that soil application of the fertilizer at the level of $2 \mathrm{ml} . \mathrm{L}^{-1}$ gave the highest means of leaves $\mathrm{N}$ level, leaves dry weight, leaves area, transplant leaves area and main stem length, which were dominated over most other treatments. Meanwhile the heighest means of leaves chlorophyll, leaves fresh weight and leaves $\mathrm{Zn}$ level were in the treatment soil application of structure at 4 $\mathrm{ml} . \mathrm{L}^{-1}$ level. In the case of the interaction between budding date and method and level of structure fertilizer, the best one was that between first budding date and soil application of the fertilizer at the level of $2 \mathrm{ml}^{-\mathrm{L}^{-1}}$, which gave the highest means of leaves $\mathrm{N}$ and $\mathrm{Zn}$ levels, leaves dry weight, leaves dry weight percentage, leaves area, leaves number, transplants leaves area and main stem length.

Keywords: Tangarin Transplants, Budding date, structure fertilizer.
\end{abstract}

Received: 18/12/2011 Accepted: 5/3/2012

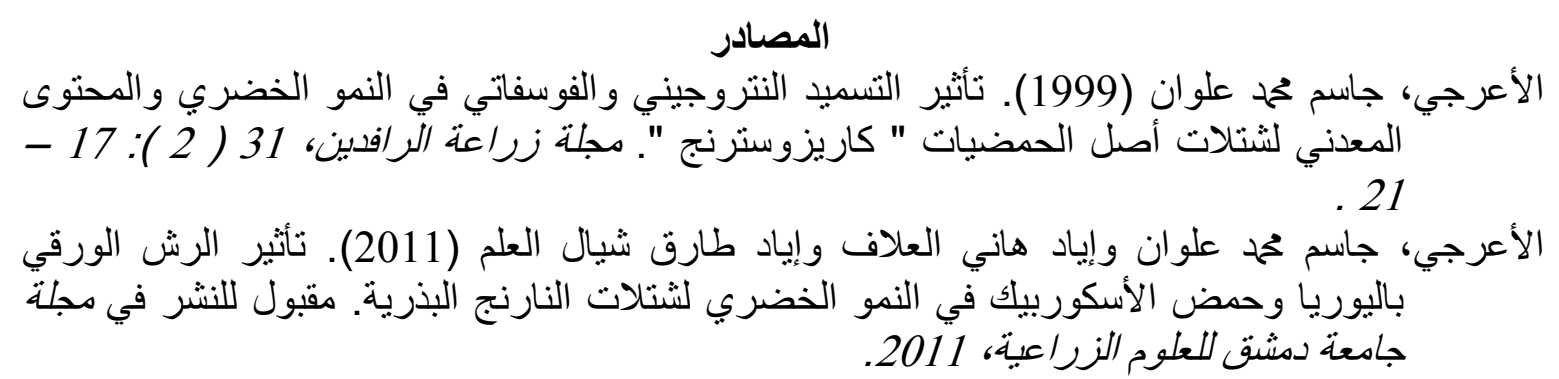


Mesopotamia J. of Agric.

Vol. (41) No. (2) 2013
ISSN:2224-9796(Online)

ISSN: 1815-316x (Print)

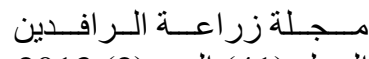

2013 المجلد (41) العدد (2)

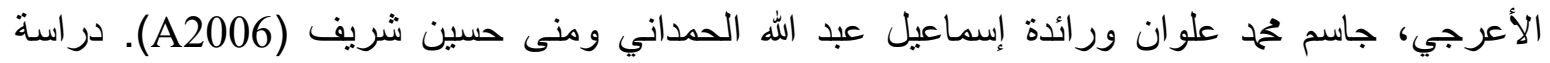

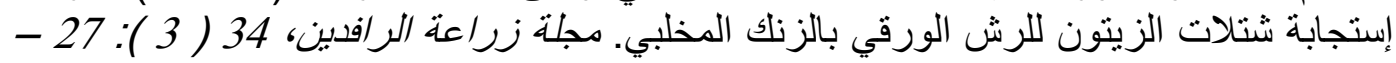

.36

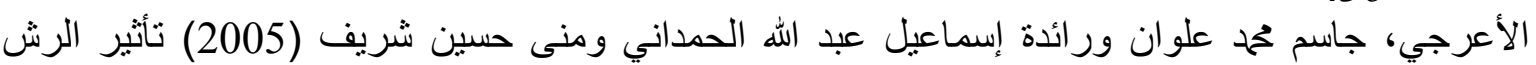

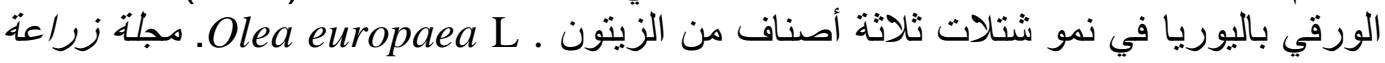
الرافدين، 33 (4): 40 - 46 - 40.

الأعرجي، جاسم ححم علوان ورائدة إسماعيل عبد الله الحمداني ونبيل محمد أمين الإمام (B 2006). تأثنير

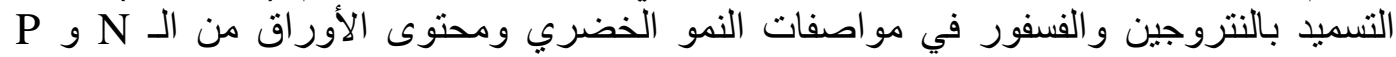

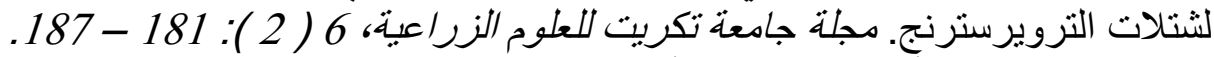

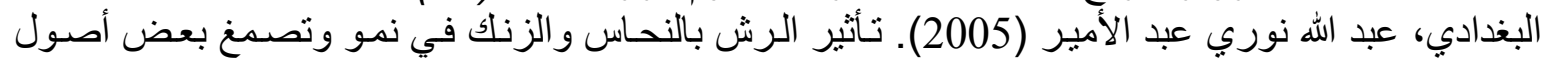

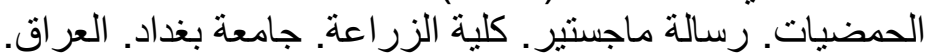

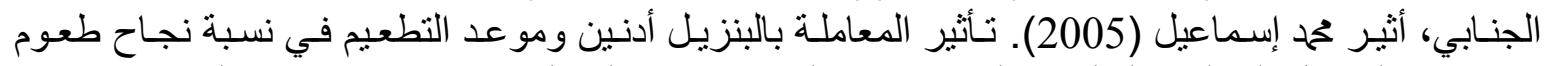

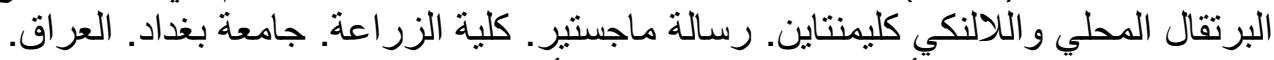

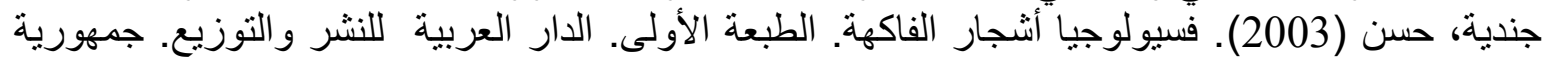

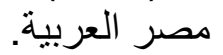

الديري، نزال وعبد العزيز ديوب ومحمد كردوش ووليد سحار (1994). بساتين الفاكهة، زر اعتها ور عايتها

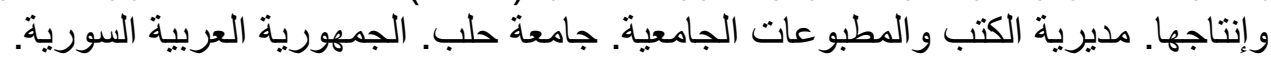

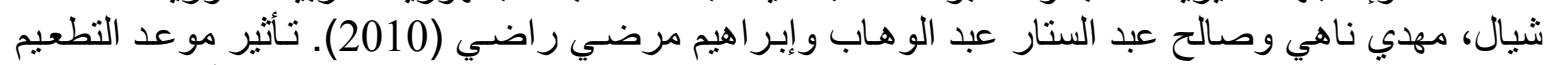

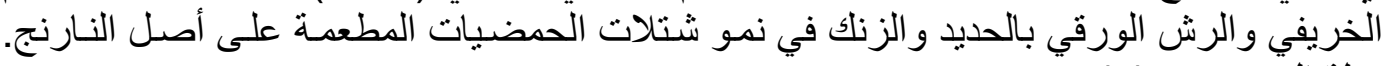

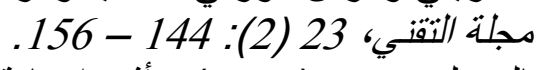

Citrus الطائي، خالد علي حسين (2008). تأثنير إضافة الكبريت والفسفور و اليوريا في نمو شتنات النارئنة aurantium L.

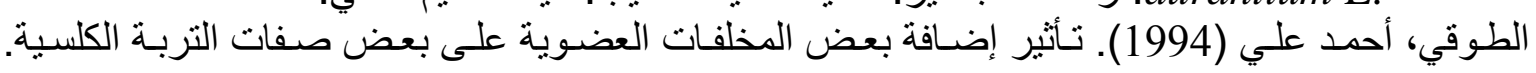

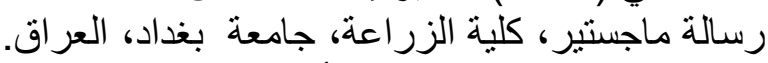

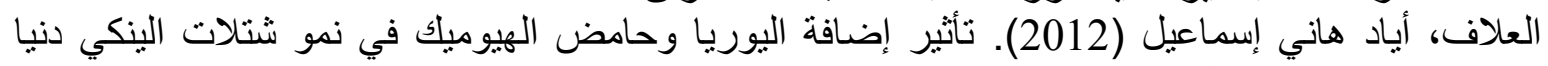

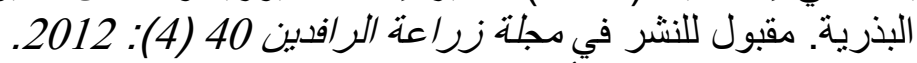

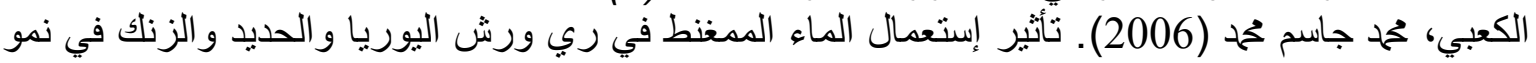

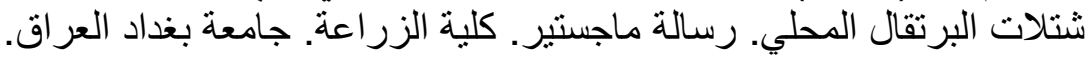

Ahmed, S. A. and A. H. Hagazi (1985). Comparative studies on autumn budding and spring budding in some citrus varieties. Agriculture Research,10 (1): $359-369$.

Anonymous (1996).Statistical Analysis System.SAS Institute Inc.Cary Nc.27511, USA.

Bal, J. S. (2005). Fruit Growing. $3^{\text {td }}$ edt. Kalyani Publishers, New Delhi- 110002.

Bhargava, B.S. and H.B. Raghupathi (1999). Analysis of plant materials for macro and micronutrients. p: 49-82 . In Tandon, H.L.S. (eds). Methods of Analysis of Soils , Plants, Water and Fertilizers . Binng Printers L- 14, Lajpat Nagor New Delhi, 110024.

Boaretto, A. E.; P. Schiavinatto; T. Muroakoand C. O. A. Bassani (1998). Efficiency of foliar and soil application of urea to young citrus plant. (C. F.).

Al - taai, K. A. H. (2008). Effect of Sulfer, Phosphorus and Urea Appending On Sour Orange Transplant Growth "Citrus aurantium”. Msc. Thesis. Commission of Technical Education. Musayab Technical Collage, In Arabic).

Boughalleb, F.; M. Mhamdi and H. Hajlaoui (2011). Response of young citrus trees 
to NPK fertilization under greenhouse and field conditions. Agriculture Journal,. 6 (3): $66-73$.

Dines, D. L.; T. S. Colvin; D. B. Taynes; T. K. Kasper; J. L. Hatfield and C. A. Cambardella (2002). Nitrogen management strategies to reduce nitrate leaching in tile drained midwesternsoils. Agronomy Journal,. 94: $71-153$.

Felixloh, J. G. and N . Bassuk (2000).Use of the minolta SPAD -502 to determine chlorophyll level in Ficus benjamina L. and populous deltoids Marsh leaf tissue .Horticulture Science, 35(3): 423.

Hassan, H. S. A.; L. F. Hagag; H. El-Wakeel; M. Abou Rawash and A. Abdel-Galel (2010). Effect of mineral, organic nitrogen fertilization and some other treatments on vegetative growth of kalamata olive young trees. Journal of American Science, 6 (2): $338-343$.

Havlin, J.L.; J.D.Beaton; S.L.Tisdale and W.L.Nelson (2005). Soil Fertility and Fertilizers $.7^{\text {th }}$ ed.Upper Saddle River, New Jersey 07458.

Obreza, T. A. (2003). Importance of potassium in a Florida citrus nutrition program. Better Crops, 87: 19 - 22.

Obreza, T. A.; M. Zekri and E. W. Hanlon (2008). Soil and leaf tissue testing. In: Nutrition Of Florida Citrus Trees, Obreza, T. A. and K. T. morgan (eds) $2^{\text {nd }}$ eds. Florida Cooperative Extension Service, Institute of Food and Agricultural Sciences, Univ. Florida, pp: 24 - 32.

Patton, L. (1984). Photosynthesis of Growth of Willow Used for Rotation. Ph.D.Dsertation submited to the Univ.of Dublin (Trinity college).

Shaheen, S. A. and A. A. Aly (2011). Response of olive cuttings to mineral fertilization and foliar sprays with urea and gibberlline. Nature \& Science, 9 (9): $76-86$.

Singh, A. (2003). Fruit Physiology and Production. Kalyani Publishers. New Delhi -110002 .

Swietlik, D. and L. Zhang (1994).Critical Zn activities for sour orange determined with chelator - buffered nutrient solution. American. Society of Horticulture. Science, 119 (4): 693 - 701. 